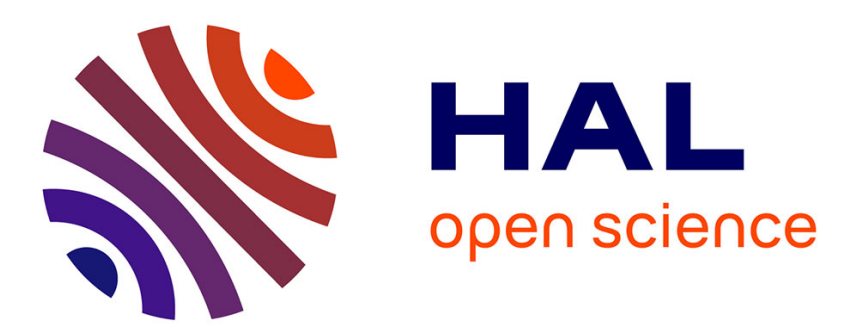

\title{
Hybrid High-Order discretizations combined with Nitsche's method for Dirichlet and Signorini boundary conditions
}

Karol L Cascavita, Franz Chouly, Alexandre Ern

\section{- To cite this version:}

Karol L Cascavita, Franz Chouly, Alexandre Ern. Hybrid High-Order discretizations combined with Nitsche's method for Dirichlet and Signorini boundary conditions. IMA Journal of Numerical Analysis, 2020, 40 (4), pp.2189-2226. 10.1093/imanum/drz038 . hal-02016378v2

\section{HAL Id: hal-02016378 \\ https://hal.science/hal-02016378v2}

Submitted on 17 Jul 2019

HAL is a multi-disciplinary open access archive for the deposit and dissemination of scientific research documents, whether they are published or not. The documents may come from teaching and research institutions in France or abroad, or from public or private research centers.
L'archive ouverte pluridisciplinaire HAL, est destinée au dépôt et à la diffusion de documents scientifiques de niveau recherche, publiés ou non, émanant des établissements d'enseignement et de recherche français ou étrangers, des laboratoires publics ou privés. 


\title{
Hybrid High-Order discretizations combined with Nitsche's method for Dirichlet and Signorini boundary conditions*
}

\author{
Karol Cascavita ${ }^{\ddagger} \quad$ Franz Chouly ${ }^{\dagger} \quad$ Alexandre Ern ${ }^{\ddagger}$
}

\begin{abstract}
We present two primal methods to weakly discretize (linear) Dirichlet and (nonlinear) Signorini boundary conditions in elliptic model problems. Both methods support polyhedral meshes with nonmatching interfaces and are based on a combination of the Hybrid High-Order (HHO) method and Nitsche's method. Since HHO methods involve both cell unknowns and face unknowns, this leads to different formulations of Nitsche's consistency and penalty terms, either using the trace of the cell unknowns (cell version) or using directly the face unknowns (face version). The face version uses equal order polynomials for cell and face unknowns, whereas the cell version uses cell unknowns of one order higher than the face unknowns. For Dirichlet conditions, optimal error estimates are established for both versions. For Signorini conditions, optimal error estimates are proven only for the cell version. Numerical experiments confirm the theoretical results, and also reveal optimal convergence for the face version applied to Signorini conditions.
\end{abstract}

Keywords. General meshes; Arbitrary order; Hybrid discretization; Dirichlet conditions; Signorini conditions; Nitsche's method.

Mathematics Subject Classification. 65N12, 65N30, 74M15.

\section{Introduction}

Hybrid Higher Order (HHO) methods have been introduced for linear elasticity in [29] and for linear diffusion problems in [30]. HHO methods are formulated in terms of face unknowns which are polynomials of arbitrary order $k \geq 0$ on each mesh face and in terms of cell unknowns which are polynomials of order $l \in\{k, k \pm 1\}$, with $l \geq 0$, in each mesh cell. The devising of HHO methods hinges on two ingredients, both defined locally in each mesh cell: a reconstruction operator and a stabilization operator. The cell unknowns can be eliminated locally by static condensation leading to a global transmission problem posed solely in terms of the face unknowns. HHO methods offer various assets: they support polyhedral meshes, lead to local conservation principles, and their construction is independent of the space dimension. Lowest-order HHO methods are closely related to Hybrid Finite Volume and Mimetic Finite Difference methods [50, 11, 34, 8]. HHO methods have been bridged in [25] to hybridizable discontinuous Galerkin methods [24] and to nonconforming Virtual Element methods [4]. HHO methods have been extended to many other PDEs, such as advection-diffusion [31] and Strokes [32] in the linear case, and Leray-Lions [27], nonlinear elasticity [9], elastoplasticity [2] with small deformations, hyperelasticity with finite deformations [1], and viscoplastic flows [16] in the nonlinear case.

The main goal of this work is to extend HHO methods to treat nonlinear Signorini boundary conditions in elliptic model problems. Signorini conditions are the constitutive building block to model unilateral contact between a deformable body and a rigid support, or between deformable bodies. Moreover, these conditions appear naturally as the Karush-Kuhn-Tucker conditions in the context of variational inequalities whenever some convex functional is minimized under some inequality constraint at the boundary. Signorini conditions also represent the first step toward more comprehensive models in computational mechanics

\footnotetext{
${ }^{1}$ F. Chouly thanks S. Natararajan and S. Bordas for the rewarding discussions on numerical methods for polyhedral meshes. K. Cascavita has benefited from a French government Grant managed by ANR within the framework of the National Program Investments for the Future ANR-11-LABX-0022-01.

${ }^{2}$ Université Bourgogne Franche-Comté, Institut de Mathématiques de Bourgogne, 21078 Dijon, France

${ }^{3}$ Université Paris-Est, CERMICS (ENPC), 77455 Marne-la-Vallée cedex 2, France and INRIA Paris, 75589 Paris, France
} 
including friction. Contact and friction problems are relevant to a broad range of applications [48, 38, 43, 40, 52, 66, 65]. Many different solutions have been proposed in the literature to enforce Signorini conditions at the discrete level, for instance in the context of finite elements. Among them, the standard finite element method (FEM) consists in a direct approximation of the variational inequality. For this standard FEM, and for many variants such as mixed/nonconforming methods [44, 5, 51], stabilized mixed methods [45], penalty methods [18], it has been quite challenging to establish optimal convergence in the $H^{1}$-norm in the case the solution belongs to $H^{1+s}(\Omega)$ with, e.g., $s \in\left(\frac{1}{2}, 1\right]$. The first fully optimal result without extra assumptions for the standard FEM has been achieved only recently in [35]. The first analyses in the 1970s were actually sub-optimal with a convergence rate of order $O\left(h^{\frac{1}{2}+\frac{s}{2}}\right)[58,42,43]$. We refer to, e.g., [47, 65, 46, 35] for more detailed reviews on the subject.

An important advance to discretize contact conditions (and more generally friction conditions) has been accomplished recently in $[19,20,21]$ by combining Nitsche's method with FEM. Nitsche's method was orginally proposed in [55] (see also $[59,41]$ ) to treat linear Dirichlet boundary conditions in a weak sense, with appropriate consistent terms that involve only the primal variables. Nitsche's method differs from standard penalty techniques which are generally not consistent [48]. Moreover, no additional unknown (Lagrange multiplier) is needed and, therefore, no discrete inf-sup condition must be fulfilled, contrary to mixed methods [43, 65]. For the Nitsche-FEM, optimal convergence in the $H^{1}(\Omega)$-norm of order $O\left(h^{s}\right)$ has been proved, provided the solution has the regularity $H^{1+s}(\Omega)$ with $s \in\left(\frac{1}{2}, k\right]$, where $k \geq 1$ is the polynomial degree of the Lagrange finite elements. To this purpose, there is no need of any additional assumption on the contact/friction zone, such as an increased regularity of the contact stress or a finite number of transition points between biding and nonbiding. Moreover, the error estimate remains valid in two and three dimensions, and for any polynomial degree $k \geq 1$ used in the FEM.

In the present work, we devise optimally convergent discretizations of Signorini conditions based on the Hybrid High-Order (HHO) method combined with Nitsche's method (Nitsche-HHO). The crucial advantage of Nitsche-HHO with respect to Nitsche-FEM is the possibility to handle polyhedral meshes. This possibility has been illustrated in recent works that deal with Nitsche's technique combined with polyhedral discretization methods to treat Dirichlet boundary conditions on curved boundaries: see [6] for the Virtual Element method and [13] for HHO methods. In addition, some polyhedral discretizations of contact problems have been proposed very recently using for instance the Virtual Element method [67, 60, 61], weak Galerkin schemes [39], hybridizable Discontinous Galerkin methods [69] and the Scaled Boundary Finite Element method [68]. In [67], contact is taken into account using either Lagrange multipliers or a penalty technique, whereas a node-to-node method is designed in [68]. Optimal convergence of order $O(h)$ for linear elements is established in the case of bilateral contact with Tresca friction in [60], with an assumption of extra regularity of the trace of the solution on the friction boundary. Note also that some other methods, proposed earlier for contact, may be suited for polyhedral discretization, for instance some discontinuous Galerkin schemes [33, 62, 63]. In [63], optimal convergence in $O(h)$ is proven for low-order schemes and a Signorini problem, under extra assumptions on the contact boundary. The use of polyhedral discretizations for contact has been motivated by an increased flexibility on meshing of the contact interfaces, and the numerical results illustrating flexibility and accuracy.

Before considering Signorini conditions, and to exemplify the main ideas in the devising and analysis of Nitsche-HHO, we first deal with the linear case of Dirichlet boundary conditions. For both Dirichlet and Signorini conditions, the formulation of Nitsche's terms in combination with HHO methods leads to different schemes according to the choice of the unknown to be used in the writing of the boundary terms: (i) the face unknown, for which we design an equal-order method, where the face and cell unknowns are of the same order; (ii) the trace of the cell unknown, for which we need a mixed-order method, where the cell unknowns are of one order higher than the face unknowns. In what follows, we refer to these variants as the face version and the cell version of Nitsche-HHO, respectively. The devising of the cell version elaborates on the idea of modifying the local reconstruction operator, as proposed in [13] in the different context of geometrically unfitted methods. Our main results are on the one hand Theorem 4.7 and Theorem 5.5 which establish the optimal convergence of Nitsche-HHO for Dirichlet conditions, using face and cell versions, respectively, and on the other hand Theorem 6.4 which establishes the optimal convergence of the cell version of Nitsche-HHO for Signorini conditions. As for Nitsche-FEM, our proofs are valid in two and three dimensions and any polynomial degree $k \geq 0$, and do not require any extra assumption apart from suitable Sobolev regularity of the exact solution. To our knowledge, this is notably the first optimal error estimate of such generality for Signorini conditions using polyhedral methods. The face version can also be extended 
to Signorini conditions and indeed delivers optimally convergent results according to our simulations, but the proof of an optimal error estimate is still ongoing.

This paper is organized as follows. We briefly present the model problems in Section 2. We introduce the discrete setting in Section 3, and we also recall some useful analysis tools. Then, we present the NitscheHHO methods for Dirichlet conditions in Section 4 and in Section 5, where we use the face version and the cell version, respectively. In Section 6, we extend the cell version of Nitsche-HHO to Signorini conditions. Finally, numerical results are presented in Section 7.

\section{Model problems}

Let $\Omega$ be a polygon/polyhedron in $\mathbb{R}^{d}, d \in\{2,3\}$, with boundary $\partial \Omega$ and unit outward normal vector $\boldsymbol{n}$. The inner product (resp. norm) in the Lebesgue space $L^{2}(\Omega)$ is denoted by $(\cdot, \cdot)_{\Omega}$ (resp. $\left.\|\cdot\|_{\Omega}\right)$. We denote by $H^{s}(\Omega), s>0$, the Sobolev spaces with inner product (resp. norm) denoted by $(\cdot, \cdot)_{s, \Omega}\left(\operatorname{resp} .\|\cdot\|_{s, \Omega}\right)$. Let $f: \Omega \rightarrow \mathbb{R}$ be a source term; we assume that $f \in L^{2}(\Omega)$. For a smooth enough function $v \in H^{s}(\Omega)$, $s>\frac{3}{2}$, we use the following notation:

$$
\sigma_{n}(v)=\boldsymbol{n} \cdot \nabla v \quad \text { on } \partial \Omega
$$

\subsection{Linear problem: Dirichlet(-Neumann) conditions}

The boundary $\partial \Omega$ is partitioned into two mutually disjoint subsets:

$$
\partial \Omega=\overline{\Gamma_{D}} \cup \overline{\Gamma_{N}}
$$

where the boundary condition is respectively a Dirichlet and a Neumann condition. We assume that $\Gamma_{D}$ has nonempty relative interior. Let us consider Dirichlet data $g_{D} \in H^{\frac{1}{2}}(\partial \Omega)$ restricted to $\Gamma_{D}$ and Neumann data $g_{N} \in L^{2}\left(\Gamma_{N}\right)$. The Poisson model problem with mixed Dirichlet-Neumann conditions reads as follows:

$$
\begin{aligned}
\Delta u+f & =0 & & \text { in } \Omega, \\
u & =g_{D} & & \text { on } \Gamma_{D}, \\
\sigma_{n}(u) & =g_{N} & & \text { on } \Gamma_{N} .
\end{aligned}
$$

The conforming Nitsche-FEM discretization of the model problem (2.1) is as follows:

$$
\left\{\begin{array}{l}
\text { Find } u_{h} \in V_{h} \text { such that } \\
a_{h}\left(u_{h}, w_{h}\right)=\ell_{h}\left(w_{h}\right) \quad \forall w_{h} \in V_{h},
\end{array}\right.
$$

where $V_{h}:=\left\{v_{h} \in C^{0}(\bar{\Omega}) \mid v_{h \mid T} \in \mathbb{P}^{k}(T) ; \forall T \in \mathcal{T}_{h}\right\}, k \geq 1$ is the polynomial degree, $\mathcal{T}_{h}$ is a member of a shape-regular sequence of meshes of $\Omega$, and the bilinear and linear forms are defined as follows:

$$
\begin{aligned}
a_{h}\left(v_{h}, w_{h}\right) & =\left(\nabla v_{h}, \nabla w_{h}\right)_{\Omega}-\int_{\Gamma_{D}}\left(\sigma_{n}\left(v_{h}\right) w_{h}+\theta v_{h} \sigma_{n}\left(w_{h}\right)\right)+\int_{\Gamma_{D}} \gamma v_{h} w_{h}, \\
\ell_{h}\left(w_{h}\right) & =\left(f, w_{h}\right)_{\Omega}-\int_{\Gamma_{D}} g_{D}\left(\theta \sigma_{n}\left(w_{h}\right)-\gamma w_{h}\right)+\int_{\Gamma_{N}} g_{N} w_{h} .
\end{aligned}
$$

Here, the user-dependent parameters are the symmetry parameter $\theta \in\{-1,0,1\}$ and the penalty parameter $\gamma$, that scales as $\gamma=\gamma_{0} h^{-1}$ with $\gamma_{0}$ taken large enough to ensure coercivity (the minimum value depends on a discrete trace inequality and therefore on the shape-regularity of the mesh sequence and the polynomial degree $k$, see, e.g., $[55,59,41])$.

Remark 2.1. The Nitsche-based FEM (2.2) encompasses symmetric and nonsymmetric variants depending upon the parameter $\theta$. The symmetric case of [55] is recovered when $\theta=1$. For the skew-symmetric variant $\theta=-1$, the well-posedness of the discrete formulation and the optimal convergence are preserved irrespectively of the value of the penalty parameter $\gamma$, that can even be taken as 0 (penalty-free Nitsche's method, see, e.g., [7, 12]). In the context of discontinuous Galerkin methods, such nonsymmetric variants are well-known as well (see, e.g., [57]). 


\subsection{Nonlinear problem: Signorini conditions}

Let us now consider a partition of the boundary $\partial \Omega$ into three mutually disjoint subsets:

$$
\Omega=\overline{\Gamma_{D}} \cup \overline{\Gamma_{N}} \cup \overline{\Gamma_{S}}
$$

where the boundary condition is respectively a Dirichlet, a Neumann, and a Signorini condition. We assume that $\Gamma_{D}$ has nonempty relative interior, and for simplicity, we consider a homogeneous Dirichlet boundary condition on $\Gamma_{D}$. The model problem reads as follows:

$$
\begin{aligned}
\Delta u+f & =0 & & \text { in } \Omega, \\
u & =0 & & \text { on } \Gamma_{D}, \\
\sigma_{n}(u) & =g_{N} & & \text { on } \Gamma_{N},
\end{aligned}
$$

and

$$
u \leq 0, \quad \sigma_{n}(u) \leq 0, \quad u \sigma_{n}(u)=0, \quad \text { on } \Gamma_{S} .
$$

It is well-known that the model problem (2.4)-(2.5) is well-posed [37].

The key idea to devise the Nitsche-FEM discretization of (2.4)-(2.5) is to reformulate the Signorini conditions (2.5) as a single nonlinear equation on the normal flux $\sigma_{n}(u)$. For any real number $x$, let $[x]_{\mathbb{R}^{-}}:=\min (x, 0)$ denote its projection onto the closed convex subset $\mathbb{R}^{-}=(-\infty, 0]$. Let us recall from [26] and [17, Prop. 2.4] that the Signorini conditions (2.5) are equivalent to

$$
\sigma_{n}(u)=\left[\phi_{\gamma}(u)\right]_{\mathbb{R}^{-}},
$$

with the notation

$$
\phi_{\gamma}(v):=\sigma_{n}(v)-\gamma v,
$$

for any smooth function $v: \Omega \rightarrow \mathbb{R}$. This leads to the following conforming Nitsche-FEM discretization of the model problem (2.4)-(2.5) [21]:

$$
\left\{\begin{array}{l}
\text { Find } u_{h} \in V_{h, D} \text { such that } \\
a_{h}\left(u_{h} ; w_{h}\right)=\ell\left(w_{h}\right) \quad \forall w_{h} \in V_{h, D},
\end{array}\right.
$$

where $V_{h, D}:=\left\{v_{h} \in V_{h} \mid v_{h \mid \Gamma_{D}}=0\right\}, V_{h}$ being defined above, and the semilinear and linear forms are defined as follows:

$$
\begin{aligned}
& a_{h}\left(v_{h} ; w_{h}\right)=\left(\nabla v_{h}, \nabla w_{h}\right)_{\Omega}-\int_{\Gamma_{S}} \frac{\theta}{\gamma} \sigma_{n}\left(v_{h}\right) \sigma_{n}\left(w_{h}\right)+\int_{\Gamma_{S}} \frac{1}{\gamma}\left[\phi_{\gamma}\left(v_{h}\right)\right]_{\mathbb{R}^{-}} \phi_{\theta \gamma}\left(w_{h}\right), \\
& \ell\left(w_{h}\right)=\left(f, w_{h}\right)_{\Omega}+\int_{\Gamma_{N}} g_{N} w_{h},
\end{aligned}
$$

with $\phi_{\theta \gamma}(v):=\theta \sigma_{n}(v)-\gamma v$. Note that $a_{h}$ is nonlinear in its first argument. The user-dependent parameters $\theta$ and $\gamma$ play the same role as in Section 2.1.

Remark 2.2. Contrarily to the case of Dirichlet boundary conditions, we can not set $\gamma=0$ when $\theta=-1$. Indeed, the Nitsche's reformulation of Signorini conditions [17, Prop. 2.4] requires that $\gamma>0$. Note also that a first attempt to derive a penalty-free Nitsche's method for Signorini boundary conditions has been proposed in [14].

\section{Discrete setting}

In this section, we recall the basic notions concerning meshes and we restate some important functional inequalities to be used in the stability and error analysis of the various Nitsche-HHO methods. 


\subsection{Meshes}

Let $\left(\mathcal{T}_{h}\right)_{h>0}$ be a mesh sequence, where for all $h>0$, the mesh $\mathcal{T}_{h}$ is composed of nonempty disjoint cells such that $\bar{\Omega}=\bigcup_{T \in \mathcal{T}_{h}} \bar{T}$. The mesh cells are conventionally open subsets in $\mathbb{R}^{d}$, they can have a polygonal/polyhedral shape with straight edges (if $d=2$ ) or planar faces (if $d=3$ ). The mesh sequence $\left(\mathcal{T}_{h}\right)_{h>0}$ is assumed to be shape-regular in the sense of [29]. In a nutshell, each mesh $\mathcal{T}_{h}$ admits a matching simplicial submesh $\Im_{h}$ having locally equivalent length scales to those of $\mathcal{T}_{h}$, and the mesh sequence $\left(\Im_{h}\right)_{h>0}$ is shape-regular in the usual sense of Ciarlet [22]. The meshsize is denoted $h=\max _{T \in \mathcal{T}_{h}} h_{T}$, with $h_{T}$ the diameter of the cell $T$. A closed subset $F$ of $\bar{\Omega}$ is called a mesh face if it is a subset with nonempty relative interior of some affine hyperplane $H_{F}$ and if (i) either there are two distinct mesh cells $T_{1}(F), T_{2}(F) \in \mathcal{T}_{h}$ so that $F=\partial T_{1}(F) \cap \partial T_{2}(F) \cap H_{F}$ (and $F$ is called an interface) (ii) or there is one mesh cell $T(F) \in \mathcal{T}_{h}$ so that $F=\partial T(F) \cap \Gamma \cap H_{F}$ (and $F$ is called a boundary face). The mesh faces are collected in the set $\mathcal{F}_{h}$ which is further partitioned into the subset of interfaces $\mathcal{F}_{h}^{\mathrm{i}}$ and the subset of boundary faces $\mathcal{F}_{h}^{\mathrm{b}}$. For all $T \in \mathcal{T}_{h}, \mathcal{F}_{\partial T}$ is the collection of the mesh faces that are subsets of $\partial T$ and $\boldsymbol{n}_{T}$ is the unit outward normal to $T$.

We use the symbol $C$ to denote a generic constant whose value can change at each occurrence as long as it is uniform with respect to the mesh size. The value can depend on the mesh regularity and the underlying polynomial degree. We abbreviate as $a \lesssim b$ the inequality $a \leq C b$ with positive real numbers $a, b$ and a constant $C>0$, whose value can change at each occurrence but is independent of the mesh size.

\subsection{Analysis tools}

Let us briefly state without proof four important technical results to be used in what follows: a discrete trace inequality on polynomials, a multiplicative trace inequality on $H^{1}$-functions, the Poincaré inequality on $H^{1}$-functions having zero mean-value and approximation properties of the $L^{2}$-orthogonal projection onto polynomials. The trace inequalities and the polynomial approximation error estimates are classical on meshes generated from a reference cell, whereas the Poincaré inequality is classical if the mesh cells are convex sets. On more general polyhedral meshes, we refer the reader to [28] and [36] for proofs.

Lemma 3.1 (Discrete trace inequality). Let $k \geq 0$ be the polynomial degree. The following holds true:

$$
\left\|v_{h}\right\|_{\partial T} \leq C_{\mathrm{dt}} h_{T}^{-\frac{1}{2}}\left\|v_{h}\right\|_{T}
$$

for all $T \in \mathcal{T}_{h}$ and all $v_{h} \in \mathbb{P}^{k}(T)$.

Lemma 3.2 (Multiplicative trace inequality). The following holds true:

$$
\|v\|_{\partial T} \leq C_{\mathrm{mt}}\left(h_{T}^{-\frac{1}{2}}\|v\|_{T}+h_{T}^{\frac{1}{2}}\|\nabla v\|_{T}\right)
$$

for all $T \in \mathcal{T}_{h}$ and all $v \in H^{1}(T)$.

Lemma 3.3 (Poincaré inequality). The following holds true:

$$
\|v\|_{T} \leq C_{\mathrm{P}} h_{T}\|\nabla v\|_{T}
$$

for all $T \in \mathcal{T}_{h}$ and all $v \in H^{1}(T)$ such that $(v, 1)_{T}=0$.

Lemma 3.4 (Polynomial approximation). Let $k \geq 0$ be the polynomial degree. Let $\pi_{T}^{k+1}$ denote the $L^{2}$ orthogonal projection onto $\mathbb{P}^{k+1}(T)$. Let $s>\frac{1}{2}$ and set $t:=\min (k+1, s)$. The following holds true:

$$
\begin{aligned}
\left\|v-\pi_{T}^{k+1}(v)\right\|_{T}+h_{T}^{\frac{1}{2}}\left\|v-\pi_{T}^{k+1}(v)\right\|_{\partial T}+h_{T} \| \nabla(v & \left.-\pi_{T}^{k+1}(v)\right) \|_{T} \\
& \quad+h_{T}^{\frac{3}{2}}\left\|\nabla\left(v-\pi_{T}^{k+1}(v)\right)\right\|_{\partial T} \leq C_{\text {app }} h_{T}^{1+t}|v|_{H^{1+t}(T)},
\end{aligned}
$$

for all $T \in \mathcal{T}_{h}$ and all $v \in H^{1+s}(T)$. The estimate (3.4) is optimal for $t=s=k+1$, in which case the right-hand side becomes $C_{\mathrm{app}} h_{T}^{2+k}|v|_{H^{2+k}(T)}$. 


\section{Dirichlet conditions: face version}

In this section, we devise and analyze the face version of the Nitsche-HHO method to approximate Dirichlet boundary conditions. We consider an equal order for the face and the cell unknowns. We assume that the meshes are compatible with the boundary partition $\partial \Omega=\overline{\Gamma_{D}} \cup \overline{\Gamma_{N}}$ from (2.1), which leads to the partition of boundary faces as $\mathcal{F}_{h}^{\mathrm{b}}=\mathcal{F}_{h}^{\mathrm{b}, \mathrm{D}} \cup \mathcal{F}_{h}^{\mathrm{b}, \mathrm{N}}$ (with obvious notation). We first present the local reconstruction and stability operators which will be used for this method, then we define the corresponding discrete Nitsche-HHO formulation and prove its well-posedness. Finally we prove its optimal convergence.

\subsection{Local reconstruction and stability operators}

Let $k \geq 0$ be the polynomial degree. For all $T \in \mathcal{T}_{h}$, the local discrete space is

$$
\widehat{U}_{T}^{k}:=\mathbb{P}^{k}(T) \times \mathbb{P}^{k}\left(\mathcal{F}_{\partial T}\right)
$$

where $\mathbb{P}^{k}(T)$ and $\mathbb{P}^{k}\left(\mathcal{F}_{\partial T}\right)$ are the spaces spanned by the restrictions to $T$ and $\mathcal{F}_{\partial T}$, respectively, of $d$ variate and piecewise $(d-1)$-variate polynomials of total degree $\leq k$. A generic element $\widehat{v}_{T} \in \widehat{U}_{T}^{k}$ is a pair $\widehat{v}_{T}=\left(v_{T}, v_{\partial T}\right)$ with $v_{T} \in \mathbb{P}^{k}(T)$ and $v_{\partial T} \in \mathbb{P}^{k}\left(\mathcal{F}_{\partial T}\right)$.

For all $T \in \mathcal{T}_{h}$, we define the local reconstruction operator $R: \widehat{U}_{T}^{k} \rightarrow \mathbb{P}^{k+1}(T)$ such that, for all $\widehat{v}_{T}=\left(v_{T}, v_{\partial T}\right) \in \widehat{U}_{T}^{k}$,

$$
\begin{aligned}
\left(\nabla R\left(\widehat{v}_{T}\right), \nabla w\right)_{T} & =\left(\nabla v_{T}, \nabla w\right)_{T}+\left(v_{\partial T}-v_{T}, \boldsymbol{n}_{T} \cdot \nabla w\right)_{\partial T} \quad \forall w \in \mathbb{P}^{k+1}(T) \\
\left(R\left(\widehat{v}_{T}\right), 1\right)_{T} & =\left(v_{T}, 1\right)_{T}
\end{aligned}
$$

which leads to a local well-posed Neumann problem that is solved by inverting the local stiffness matrix in $\mathbb{P}^{k+1}(T)$. The local stabilization operator $S: \widehat{U}_{T}^{k} \rightarrow \mathbb{P}^{k}\left(\mathcal{F}_{\partial T}\right)$ is used to penalize the difference between the face unknown $v_{\partial T}$ and the trace of the cell unknown $v_{T \mid \partial T}$ in a least-squares sense. The operator $S$ is defined such that, for all $\widehat{v}_{T}=\left(v_{T}, v_{\partial T}\right) \in \widehat{U}_{T}^{k}$,

$$
S\left(\widehat{v}_{T}\right):=\pi_{\partial T}^{k}\left(v_{\partial T}-R\left(\widehat{v}_{T}\right)_{\mid \partial T}\right)-\pi_{T}^{k}\left(v_{T}-R\left(\widehat{v}_{T}\right)\right)_{\mid \partial T},
$$

where $\pi_{T}^{k}$ and $\pi_{\partial T}^{k}$ denote the $L^{2}$-orthogonal projectors onto $\mathbb{P}^{k}(T)$ and $\mathbb{P}^{k}\left(\mathcal{F}_{\partial T}\right)$, respectively. Since $R\left(v_{T}, v_{T \mid \partial T}\right)=v_{T}$, one has $S\left(v_{T}, v_{T \mid \partial T}\right)=0$.

We use the two above operators to formulate the following local bilinear form $\widehat{a}_{T}$ on $\widehat{U}_{T}^{k} \times \widehat{U}_{T}^{k}$ that mimics locally the exact local bilinear form $(\nabla v, \nabla w)_{T}$ :

$$
\widehat{a}_{T}\left(\widehat{v}_{T}, \widehat{w}_{T}\right):=\left(\nabla R\left(\widehat{v}_{T}\right), \nabla R\left(\widehat{w}_{T}\right)\right)_{T}+\left(\eta_{\partial T} S\left(\widehat{v}_{T}\right), S\left(\widehat{w}_{T}\right)\right)_{\partial T},
$$

where $\eta_{\partial T}$ is the piecewise constant function on $\partial T$ such that $\eta_{\partial T \mid F}=h_{F}^{-1}$, for all $F \in \mathcal{F}_{\partial T}$.

We equip the discrete space $\widehat{U}_{T}^{k}$ with the following $H^{1}$-like seminorm:

$$
\left|\widehat{v}_{T}\right|_{\widehat{U}_{T}^{k}}^{2}:=\left\|\nabla v_{T}\right\|_{T}^{2}+\left\|\eta_{\partial T}^{\frac{1}{2}}\left(v_{\partial T}-v_{T \mid \partial T}\right)\right\|_{\partial T}^{2} \quad \forall \widehat{v}_{T}=\left(v_{T}, v_{\partial T}\right) \in \widehat{U}_{T}^{k}
$$

so that $\left|\widehat{v}_{T}\right|_{\widehat{U}_{T}^{k}}=0$ implies that $v_{T}=v_{\partial T}=c s t$. Let us briefly outline the stability, boundedness, and polynomial invariance properties that motivate the design of the local operators $R$ and $S$. For the proofs, we refer the reader to [30].

Lemma 4.1 (Stability and boundedness). There is a real number $\rho>0$, independent of $h$, such that, for all $T \in \mathcal{T}_{h}$ and all $\widehat{v}_{T} \in \widehat{U}_{T}^{k}$,

$$
\rho^{-1}\left|\widehat{v}_{T}\right|_{\widehat{U}_{T}^{k}}^{2} \leq\left\|\nabla R\left(\widehat{v}_{T}\right)\right\|_{T}^{2}+\left\|\eta_{\partial T}^{\frac{1}{2}} S\left(\widehat{v}_{T}\right)\right\|_{\partial T}^{2} \leq \rho\left|\widehat{v}_{T}\right|_{\widehat{U}_{T}^{k}}^{2}
$$

The parameter $\rho$ only depends on the mesh regularity and the polynomial degree. The first inequality in (4.2) implies the coercivity of the bilinear form $\widehat{a}_{T}$ on the one-dimensional subspace $\left\{\widehat{v}_{T} \in \widehat{U}_{T}^{k} ; v_{T}=v_{\partial T}=c s t\right\}$. 
Lemma 4.2 (Polynomial invariance). Let $\widehat{I}_{T}: H^{1}(T) \rightarrow \widehat{U}_{T}^{k}$ be the local reduction (or interpolation) operator such that $\widehat{I}_{T}(v)=\left(\pi_{T}^{k}(v), \pi_{\partial T}^{k}\left(v_{\mid \partial T}\right)\right) \in \widehat{U}_{T}^{k}$, for all $v \in H^{1}(T)$ and all $T \in \mathcal{T}_{h}$. Then we have

$$
R\left(\widehat{I}_{T}(v)\right)=E_{T}(v) \quad \forall v \in H^{1}(T),
$$

where $E_{T}: H^{1}(T) \rightarrow \mathbb{P}^{k+1}(T)$ is the standard local elliptic projector such that, for all $v \in H^{1}(T)$,

$$
\left(\nabla\left(E_{T}(v)-v\right), \nabla w\right)_{T}=0, \forall w \in \mathbb{P}^{k+1}(T), \quad\left(E_{T}(v)-v, 1\right)_{T}=0 .
$$

Moreover the following holds true for the local stabilization operator:

$$
S\left(\widehat{I}_{T}(p)\right)=0 \quad \forall p \in \mathbb{P}^{k+1}(T) .
$$

We will also need the following approximation result for the local elliptic projector and for the stabilization operator.

Lemma 4.3 (Approximation). Let $s>\frac{1}{2}$ and set $t:=\min (k+1, s)$. There is a uniform constant $C$ such that the following holds true for all $T \in \mathcal{T}_{h}$ and all $v \in H^{1+s}(T)$ :

$$
\begin{aligned}
\left\|v-E_{T}(v)\right\|_{T}+h_{T}^{\frac{1}{2}}\left\|v-E_{T}(v)\right\|_{\partial T}+h_{T} \| \nabla\left(v-E_{T}(v)\right) & \|_{T} \\
& +h_{T}^{\frac{3}{2}}\left\|\nabla\left(v-E_{T}(v)\right)\right\|_{\partial T} \leq C h_{T}^{1+s}|v|_{H^{1+s}(T)} .
\end{aligned}
$$

Moreover, for all $T \in \mathcal{T}_{h}$ and all $v \in H^{1}(T)$, we have

$$
\left\|\eta_{\partial T}^{\frac{1}{2}} S\left(\widehat{I}_{T}(v)\right)\right\|_{\partial T} \leq C\left\|\nabla\left(v-E_{T}(v)\right)\right\|_{T} .
$$

Proof. See [30, Lemma 3] for (4.6) (the proof uses the approximation result from Lemma 3.4). Concerning (4.7), we proceed as in [30, Eq. (45)]. Owing to the definition of $S$ and since $E_{T}=R \circ \widehat{I}_{T}$ (see (4.3)), we have

$$
S\left(\widehat{I}_{T}(v)\right)=\pi_{\partial T}^{k}\left(v-E_{T}(v)_{\mid \partial T}\right)-\pi_{T}^{k}\left(v-E_{T}(v)\right)_{\mid \partial T} .
$$

We then use the triangle inequality, the stability of the $L^{2}$-projectors, that $\eta_{\partial T}$ is piecewise constant, the regularity of the mesh sequence, and the discrete trace inequality from Lemma 3.1 to infer that (the value of $C$ can change at each occurrence)

$$
\begin{aligned}
\left\|\eta_{\partial T}^{\frac{1}{2}} S\left(\widehat{I}_{T}(v)\right)\right\|_{\partial T} & \leq\left\|\eta_{\partial T}^{\frac{1}{2}} \pi_{\partial T}^{k}\left(v-E_{T}(v)\right)\right\|_{\partial T}+\left\|\eta_{\partial T}^{\frac{1}{2}} \pi_{T}^{k}\left(v-E_{T}(v)\right)\right\|_{\partial T} \\
& \leq\left\|\eta_{\partial T}^{\frac{1}{2}}\left(v-E_{T}(v)\right)\right\|_{\partial T}+C h_{T}^{-1}\left\|\pi_{T}^{k}\left(v-E_{T}(v)\right)\right\|_{T} \\
& \leq C h_{T}^{-1}\left(h_{T}^{\frac{1}{2}}\left\|v-E_{T}(v)\right\|_{\partial T}+\left\|v-E_{T}(v)\right\|_{T}\right) .
\end{aligned}
$$

To conclude, we invoke the multiplicative trace inequality from Lemma 3.2 and the local Poincaré inequality from Lemma 3.3 (the function $v-E_{T}(v)$ has, by construction, zero mean-value in $T$ ).

\subsection{Discrete problem, stability, and well-posedness}

The global discrete space for the face version of the Nitsche-HHO method is defined to be

$$
\widehat{U}_{h}^{k}:=\mathbb{P}^{k}\left(\mathcal{T}_{h}\right) \times \mathbb{P}^{k}\left(\mathcal{F}_{h}\right),
$$

with the notation $\widehat{v}_{h}=\left(\left(v_{T}\right)_{T \in \mathcal{T}_{h}},\left(v_{F}\right)_{F \in \mathcal{F}_{h}}\right)$ for a generic element $\widehat{v}_{h} \in \widehat{U}_{h}^{k}$. For all $T \in \mathcal{T}_{h}$, we denote by $\widehat{v}_{T}=\left(v_{T}, v_{\partial T}\right) \in \widehat{U}_{T}^{k}$ the components of $\widehat{v}_{h}$ attached to the mesh cell $T$ and the faces composing its boundary.

As in the conforming Nitsche-FEM (2.2), we consider a symmetry parameter $\theta \in\{-1,0,1\}$ and a penalty parameter $\gamma>0$ that will be taken of the form $\gamma_{\mid F}=\gamma_{0} h_{F}^{-1}$, for all $F \in \mathcal{F}_{h}^{\mathrm{b}, \mathrm{D}}$, with $\gamma_{0}$ large enough (depending on the constant $C_{\mathrm{dt}}$ from Lemma 3.1). The discrete Nitsche-HHO problem is as follows:

$$
\left\{\begin{array}{l}
\text { Find } \widehat{u}_{h} \in \widehat{U}_{h}^{k} \text { such that } \\
\widehat{a}_{h}\left(\widehat{u}_{h}, \widehat{w}_{h}\right)=\widehat{\ell}_{h}\left(\widehat{w}_{h}\right) \quad \forall \widehat{w}_{h} \in \widehat{U}_{h}^{k} .
\end{array}\right.
$$


For all $\widehat{v}_{h}, \widehat{w}_{h} \in \widehat{U}_{h}^{k}$, the global discrete bilinear form $\widehat{a}_{h}$ and the global discrete linear form $\widehat{\ell}_{h}$ are defined respectively by (compare with $(2.3)$ )

$$
\begin{aligned}
\widehat{a}_{h}\left(\widehat{v}_{h}, \widehat{w}_{h}\right):= & \sum_{T \in \mathcal{T}_{h}} \widehat{a}_{T}\left(\widehat{v}_{T}, \widehat{w}_{T}\right)-\sum_{F \in \mathcal{F}_{h}^{b, D}}\left(\sigma_{n}\left(R\left(\widehat{v}_{T(F)}\right)\right), w_{F}\right)_{F} \\
& -\sum_{F \in \mathcal{F}_{h}^{b, D}} \theta\left(v_{F}, \sigma_{n}\left(R\left(\widehat{w}_{T(F)}\right)\right)\right)_{F}+\sum_{F \in \mathcal{F}_{h}^{b, D}} \gamma\left(v_{F}, w_{F}\right)_{F}, \\
\widehat{\ell}_{h}\left(\widehat{w}_{h}\right):= & \sum_{T \in \mathcal{T}_{h}}\left(f, w_{T}\right)_{T}+\sum_{F \in \mathcal{F}_{h}^{b, N}}\left(g_{N}, w_{F}\right)_{F} \\
& -\sum_{F \in \mathcal{F}_{h}^{b, D}} \theta\left(g_{D}, \sigma_{n}\left(R\left(\widehat{w}_{T(F)}\right)\right)\right)_{F}+\sum_{F \in \mathcal{F}_{h}^{b, D}} \gamma\left(g_{D}, w_{F}\right)_{F},
\end{aligned}
$$

where, for all $F \in \mathcal{F}_{h}^{\mathrm{b}}, T(F)$ is the single mesh cell of which $F$ is a boundary face. We equip the space $\widehat{U}_{h}^{k}$ with the norm

$$
\left\|\widehat{v}_{h}\right\|_{\widehat{U}_{h}^{k}}^{2}:=\sum_{T \in \mathcal{T}_{h}}\left|\widehat{v}_{T}\right|_{\widehat{U}_{T}^{k}}^{2}+\sum_{F \in \mathcal{F}_{h}^{\mathrm{b}, \mathrm{D}}} h_{F}^{-1}\left\|v_{F}\right\|_{F}^{2}, \quad \forall \widehat{v}_{h} \in \widehat{U}_{h}^{k},
$$

with the local seminorms $|\cdot|_{\widehat{U}_{T}^{k}}$ defined in (4.1). Since the subset $\mathcal{F}_{h}^{\mathrm{b}, \mathrm{D}}$ is nonempty (recall that $\Gamma_{D}$ has nonempty relative interior), it is readily verified that $\|\cdot\|_{\widehat{U}_{h}^{k}}$ defines a norm on $\widehat{U}_{h}^{k}$.

Let us now address the well-posedness of the discrete problem (4.8). For brevity, we only present the proof for the choice $\theta=1$ of the symmetry parameter. Well-posedness also holds true for $\theta=0$ (with a less stringent lower bound on $\gamma_{0}$ ) and for $\theta=-1$ (with the simple requirement that $\gamma_{0}>0$ ). Let $n^{\mathrm{b}, \mathrm{D}}$ be the maximum number of faces in $\mathcal{F}_{h}^{\mathrm{b}, \mathrm{D}}$ that a mesh cell can have ( $n^{\mathrm{b}, \mathrm{D}} \leq d$ on simplicial meshes).

Lemma 4.4 (Coercivity and well-posedness). Assume that $\theta=1$ and that $\gamma_{0}>2 n^{\mathrm{b}, \mathrm{D}} C_{\mathrm{dt}}^{2}$, where $C_{\mathrm{dt}}$ results from the discrete trace inequality of Lemma 3.1. Let us set the penalty parameter to $\gamma_{\mid F}:=\gamma_{0} h_{F}^{-1}$, for all $F \in \mathcal{F}_{h}^{\mathrm{b}, \mathrm{D}}$. Then the discrete bilinear form $\widehat{a}_{h}$ is coercive, and the discrete problem (4.8) is well-posed.

Proof. It suffices to prove coercivity since well-posedness then follows from the Lax-Milgram Lemma. Let $\widehat{v}_{h} \in \widehat{U}_{h}^{k}$. Using the discrete trace inequality from Lemma 3.1, we have

$$
\begin{aligned}
\sum_{F \in \mathcal{F}_{h}^{b, D}} 2\left(\sigma_{n}\left(R\left(\widehat{v}_{T(F)}\right)\right), v_{F}\right)_{F} & \geq-\sum_{F \in \mathcal{F}_{h}^{b, D}}\left(n^{\mathrm{b}, \mathrm{D}}\right)^{-\frac{1}{2}} C_{\mathrm{dt}}^{-1} h_{F}^{\frac{1}{2}}\left\|\nabla R\left(\widehat{v}_{T(F)}\right)\right\|_{F} \times 2\left(n^{\mathrm{b}, \mathrm{D}}\right)^{\frac{1}{2}} C_{\mathrm{dt}} h_{F}^{-\frac{1}{2}}\left\|v_{F}\right\|_{F} \\
& \geq-\sum_{T \in \mathcal{T}_{h}^{\mathrm{b}, \mathrm{D}}} \frac{1}{2}\left\|\nabla R\left(\widehat{v}_{T}\right)\right\|_{T}^{2}-\sum_{F \in \mathcal{F}_{h}^{b, D}} 2 n^{\mathrm{b}, \mathrm{D}} C_{\mathrm{dt}}^{2} h_{F}^{-1}\left\|v_{F}\right\|_{F}^{2},
\end{aligned}
$$

where $\mathcal{T}_{h}^{\mathrm{b}, \mathrm{D}}$ is the collection of the mesh cells having a boundary face in $\mathcal{F}_{h}^{\mathrm{b}, \mathrm{D}}$. Bounding the first summation by the summation over all the mesh cells, we infer that

$$
\begin{aligned}
\widehat{a}_{h}\left(\widehat{v}_{h}, \widehat{v}_{h}\right) & \geq \sum_{T \in \mathcal{T}_{h}} \frac{1}{2}\left\|\nabla R\left(\widehat{v}_{T}\right)\right\|_{T}^{2}+\sum_{T \in \mathcal{T}_{h}}\left\|\eta_{\partial T}^{\frac{1}{2}} S\left(\widehat{v}_{T}\right)\right\|_{\partial T}^{2}+\left(\gamma_{0}-2 n^{\mathrm{b}, \mathrm{D}} C_{\mathrm{dt}}^{2}\right) \sum_{F \in \mathcal{F}_{h}^{\mathrm{b}, \mathrm{D}}} h_{F}^{-1}\left\|v_{F}\right\|_{F}^{2} \\
& \geq \min \left(\frac{1}{2} \rho^{-1}, \gamma_{0}-2 n^{\mathrm{b}, \mathrm{D}} C_{\mathrm{dt}}^{2}\right)\left\|\widehat{v}_{h}\right\|_{\widehat{U}_{h}^{k}}^{2} .
\end{aligned}
$$

This concludes the proof.

Remark 4.5 (Choosing the symmetry parameter $\theta$ ). (i) For $\theta=1$, one obtains a discrete problem with variational structure, that is, the discrete problem takes the form of the Euler equations characterizing the minimizer of the convex functional

$$
\mathcal{J}_{h}\left(\widehat{w}_{h}\right)=\frac{1}{2} \widehat{a}_{h}\left(\widehat{w}_{h}, \widehat{w}_{h}\right)-\widehat{\ell}_{h}\left(\widehat{w}_{h}\right)
$$

defined for every $\widehat{w}_{h} \in \widehat{U}_{h}^{k}$. (ii) For $\theta=0$, one recovers a simpler method since some terms in the formulation vanish, and the lower bound on $\gamma_{0}$ becomes $\gamma_{0}>\frac{1}{2} n^{\mathrm{b}, \mathrm{D}} C_{\mathrm{dt}}^{2}$. (iii) For $\theta=-1$, the stability properties of the method are stronger since it suffices to take $\gamma_{0}>0$. 


\subsection{Error analysis}

The first important step in the error analysis is to bound the consistency error which is defined as follows:

$$
\mathcal{E}_{h}\left(\widehat{w}_{h}\right):=\widehat{\ell}_{h}\left(\widehat{w}_{h}\right)-\widehat{a}_{h}\left(\widehat{I}_{h}(u), \widehat{w}_{h}\right), \quad \forall \widehat{w}_{h} \in \widehat{U}_{h}^{k},
$$

where the global reduction operator $\widehat{I}_{h}: H^{1}(\Omega) \rightarrow \widehat{U}_{h}^{k}$ is defined such that the local components of $\widehat{I}_{h}(v)$, for all $v \in H^{1}(\Omega)$, attached to a mesh cell $T \in \mathcal{T}_{h}$, are $\widehat{I}_{T}\left(v_{\mid T}\right)$ (this definition is meaningful since a function in $H^{1}(\Omega)$ is single-valued at all the mesh interfaces). As above, we only give proofs for $\theta=1$; the proofs for the other values $\theta \in\{-1,0\}$ follow by minor adaptations of the arguments for $\theta=1$.

Lemma 4.6 (Consistency). Assume that $\theta=1$ and that $u \in H^{1+s}(\Omega)$ with $s>\frac{1}{2}$. The following holds true:

$$
\left|\mathcal{E}_{h}\left(\widehat{w}_{h}\right)\right| \lesssim\left(\sum_{T \in \mathcal{T}_{h}}\left\|u-E_{T}(u)\right\|_{\sharp, T}^{2}\right)^{\frac{1}{2}}\left\|\widehat{w}_{h}\right\|_{\widehat{U}_{h}^{k}}, \quad \forall \widehat{w}_{h} \in \widehat{U}_{h}^{k},
$$

with $\|v\|_{\sharp, T}^{2}:=\|\nabla v\|_{T}^{2}+h_{T}\left\|\boldsymbol{n}_{T} \cdot \nabla v\right\|_{\partial T}^{2}$ for any function $v \in H^{1+s}(T), s>\frac{1}{2}$, and all $T \in \mathcal{T}_{h}$.

Proof. Let $\widehat{w}_{h} \in \widehat{U}_{h}^{k}$. Let us introduce the shorthand notation

$$
\eta_{\gamma}\left(\widehat{w}_{T(F)}\right):=-\sigma_{n}\left(R\left(\widehat{w}_{T(F)}\right)\right)+\gamma w_{F} .
$$

Using the definitions (4.9a)-(4.9b) of $\widehat{a}_{h}, \widehat{\ell}_{h}$, the PDE and the boundary conditions satisfied by the exact solution $u$, and since $R \circ \widehat{I}_{T}=E_{T}$ (see (4.3)), we have

$$
\begin{aligned}
\mathcal{E}_{h}\left(\widehat{w}_{h}\right)= & \sum_{T \in \mathcal{T}_{h}}\left(-\Delta u, w_{T}\right)_{T}+\sum_{F \in \mathcal{F}_{h}^{b, N}}\left(\sigma_{n}(u), w_{F}\right)_{F}+\sum_{F \in \mathcal{F}_{h}^{b, D}}\left(u, \eta_{\gamma}\left(\widehat{w}_{T(F)}\right)\right)_{F} \\
& -\sum_{T \in \mathcal{T}_{h}}\left(\nabla E_{T}(u), \nabla R\left(\widehat{w}_{T}\right)\right)_{T}-\sum_{T \in \mathcal{T}_{h}}\left(\eta_{\partial T} S\left(\widehat{I}_{T}(u)\right), S\left(\widehat{w}_{T}\right)\right)_{\partial T} \\
& +\sum_{F \in \mathcal{F}_{h}^{b, D}}\left(\sigma_{n}\left(E_{T(F)}(u)\right), w_{F}\right)_{F}-\left(\pi_{F}^{k}(u), \eta_{\gamma}\left(\widehat{w}_{T(F)}\right)\right)_{F} .
\end{aligned}
$$

Since $\eta_{\gamma}\left(\widehat{w}_{T(F)}\right)$ is a polynomial of degree at most $k$ on each boundary face in $\mathcal{F}_{h}^{\mathrm{b}, \mathrm{D}}$, we infer that $\left(u, \eta_{\gamma}\left(\widehat{w}_{T(F)}\right)\right)_{F}=\left(\pi_{F}^{k}(u), \eta_{\gamma}\left(\widehat{w}_{T(F)}\right)\right)_{F}$. Re-arranging terms leads to

$$
\mathcal{E}_{h}\left(\widehat{w}_{h}\right)=\mathcal{T}_{1}-\mathcal{T}_{2}-\mathcal{T}_{3},
$$

where

$$
\begin{aligned}
& \mathcal{T}_{1}=\sum_{T \in \mathcal{T}_{h}}\left(-\Delta u, w_{T}\right)_{T}+\sum_{F \in \mathcal{F}_{h}^{b, N}}\left(\sigma_{n}(u), w_{F}\right)_{F}, \\
& \mathcal{T}_{2}=\sum_{T \in \mathcal{T}_{h}}\left(\nabla E_{T}(u), \nabla R\left(\widehat{w}_{T}\right)\right)_{T}-\sum_{F \in \mathcal{F}_{h}^{b, D}}\left(\sigma_{n}\left(E_{T(F)}(u)\right), w_{F}\right)_{F}, \\
& \mathcal{T}_{3}=\sum_{T \in \mathcal{T}_{h}}\left(\eta_{\partial T} S\left(\widehat{I}_{T}(u)\right), S\left(\widehat{w}_{T}\right)\right)_{\partial T} .
\end{aligned}
$$

Integrating by parts in each mesh cell and using that $\sigma_{n}(u)$ is single-valued across all the mesh interfaces (and well-defined since $s>\frac{1}{2}$ ), we obtain

$$
\begin{aligned}
\mathcal{T}_{1} & =\sum_{T \in \mathcal{T}_{h}}\left(\nabla u, \nabla w_{T}\right)_{T}-\sum_{T \in \mathcal{T}_{h}}\left(\sigma_{n}(u), w_{T}\right)_{\partial T}+\sum_{F \in \mathcal{F}_{h}^{b, N}}\left(\sigma_{n}(u), w_{F}\right)_{F} \\
& =\sum_{T \in \mathcal{T}_{h}}\left(\nabla u, \nabla w_{T}\right)_{T}-\sum_{T \in \mathcal{T}_{h}}\left(\sigma_{n}(u), w_{T}-w_{\partial T}\right)_{\partial T}-\sum_{F \in \mathcal{F}_{h}^{b, D}}\left(\sigma_{n}(u), w_{F}\right)_{F} .
\end{aligned}
$$


Using the definition of $R\left(\widehat{w}_{T}\right)$, we infer that

$$
\mathcal{T}_{2}=\sum_{T \in \mathcal{T}_{h}}\left(\nabla E_{T}(u), \nabla w_{T}\right)_{T}-\sum_{T \in \mathcal{T}_{h}}\left(\sigma_{n}\left(E_{T}(u)\right), w_{T}-w_{\partial T}\right)_{\partial T}-\sum_{F \in \mathcal{F}_{h}^{b, D}}\left(\sigma_{n}\left(E_{T(F)}(u)\right), w_{F}\right)_{F} .
$$

Consequently, if we define for all $T \in \mathcal{T}_{h}$ the function $\delta_{T}:=u_{\mid T}-E_{T}\left(u_{\mid T}\right)$, we obtain

$$
\begin{aligned}
\mathcal{T}_{1}-\mathcal{T}_{2} & =\sum_{T \in \mathcal{T}_{h}}\left(\nabla \delta_{T}, \nabla w_{T}\right)_{T}-\sum_{T \in \mathcal{T}_{h}}\left(\sigma_{n}\left(\delta_{T}\right), w_{T}-w_{\partial T}\right)_{\partial T}-\sum_{F \in \mathcal{F}_{h}^{b, D}}\left(\sigma_{n}\left(\delta_{T(F)}\right), w_{F}\right)_{F} \\
& =-\sum_{T \in \mathcal{T}_{h}}\left(\sigma_{n}\left(\delta_{T}\right), w_{T}-w_{\partial T}\right)_{\partial T}-\sum_{F \in \mathcal{F}_{h}^{b, D}}\left(\sigma_{n}\left(\delta_{T(F)}\right), w_{F}\right)_{F},
\end{aligned}
$$

where we used that $\left(\nabla \delta_{T}, \nabla w_{T}\right)_{T}=0$ since $w_{T} \in \mathbb{P}^{k}(T) \subset \mathbb{P}^{k+1}(T)$. Invoking the Cauchy-Schwarz inequality and recalling the definition of the norm $\left\|\widehat{w}_{h}\right\|_{\widehat{U}_{h}^{k}}$, we infer that

$$
\left|\mathcal{T}_{1}-\mathcal{T}_{2}\right| \lesssim\left(\sum_{T \in \mathcal{T}_{h}}\left\|\delta_{T}\right\|_{\sharp, T}^{2}\right)^{\frac{1}{2}}\left\|\widehat{w}_{h}\right\|_{\widehat{U}_{h}^{k}}
$$

Finally, the Cauchy-Schwarz inequality, the bound (4.7) and the upper bound in (4.2) imply that

$$
\left|\mathcal{T}_{3}\right| \leq\left(\sum_{T \in \mathcal{T}_{h}}\left\|\eta_{\partial T}^{\frac{1}{2}} S\left(\widehat{I}_{T}(u)\right)\right\|_{\partial T}^{2}\right)^{\frac{1}{2}}\left(\sum_{T \in \mathcal{T}_{h}}\left\|\eta_{\partial T}^{\frac{1}{2}} S\left(\widehat{w}_{T}\right)\right\|_{\partial T}^{2}\right)^{\frac{1}{2}} \lesssim\left(\sum_{T \in \mathcal{T}_{h}}\left\|\delta_{T}\right\|_{\sharp, T}^{2}\right)^{\frac{1}{2}}\left\|\widehat{w}_{h}\right\|_{\widehat{U}_{h}^{k}} .
$$

This concludes the proof.

We now prove the optimal convergence of the method (4.8).

Theorem 4.7 ( $H^{1}$-error estimate). Assume that $u \in H^{1+s}(\Omega)$ with $s>\frac{1}{2}$. The following holds true:

$$
\sum_{T \in \mathcal{T}_{h}}\left\|\nabla\left(u-R\left(\widehat{u}_{T}\right)\right)\right\|_{T}^{2} \lesssim \sum_{T \in \mathcal{T}_{h}}\left\|u-E_{T}(u)\right\|_{\sharp, T}^{2} .
$$

Consequently, letting $t:=\min (k+1, s)$, we have

$$
\sum_{T \in \mathcal{T}_{h}}\left\|\nabla\left(u-R\left(\widehat{u}_{T}\right)\right)\right\|_{T}^{2} \lesssim \sum_{T \in \mathcal{T}_{h}} h_{T}^{2 t}|u|_{H^{2+t}(T)}^{2} .
$$

Proof. Let us set $\widehat{w}_{h}:=\widehat{u}_{h}-\widehat{I}_{h}(u) \in \widehat{U}_{h}^{k}$. The coercivity of $\widehat{a}_{h}$ from Lemma 4.4 and the bound on the consistency error from Lemma 4.6 imply that

$$
\left\|\widehat{w}_{h}\right\|_{\widehat{U}_{h}^{k}} \lesssim \frac{\widehat{a}_{h}\left(\widehat{w}_{h}, \widehat{w}_{h}\right)}{\left\|\widehat{w}_{h}\right\|_{\widehat{U}_{h}^{k}}}=\frac{\mathcal{E}_{h}\left(\widehat{w}_{h}\right)}{\left\|\widehat{w}_{h}\right\|_{\widehat{U}_{h}^{k}}} \lesssim\left(\sum_{T \in \mathcal{T}_{h}}\left\|u-E_{T}(u)\right\|_{\sharp, T}^{2}\right)^{\frac{1}{2}} .
$$

Using the upper bound from Lemma 4.1, we infer that

$$
\sum_{T \in \mathcal{T}_{h}}\left\|\nabla\left(R\left(\widehat{u}_{T}\right)-E_{T}(u)\right)\right\|_{T}^{2}=\sum_{T \in \mathcal{T}_{h}}\left\|\nabla R\left(\widehat{w}_{T}\right)\right\|_{T}^{2} \lesssim\left\|\widehat{w}_{h}\right\|_{\widehat{U}_{h}^{k}}^{2} \lesssim \sum_{T \in \mathcal{T}_{h}}\left\|u-E_{T}(u)\right\|_{\sharp, T}^{2} .
$$

The estimate (4.13) results from this bound, the triangle inequality, and the definition of $\|\cdot\|_{\sharp, T}$. Finally, the estimate (4.14) is a consequence of (4.13) and Lemma 4.3. 


\section{Dirichlet conditions: cell version}

The goal of this section is to extend our analysis to the cell version of the Nitsche-HHO method, still to approximate Dirichlet conditions. The main novelty is that the cell unknowns are now of one order higher than the face unknowns and are used in the formulation of Nitsche's consistency and penalty terms. An optimal error estimate is achieved by slightly changing the definition of the reconstruction operator. The reason for this change is somewhat subtle and will appear when bounding the consistency error.

As in the previous section, we assume that the meshes are compatible with the boundary partition $\partial \Omega=\overline{\Gamma_{D}} \cup \overline{\Gamma_{N}}$ from (2.1), which leads again to the partition of boundary faces as $\mathcal{F}_{h}^{\mathrm{b}}=\mathcal{F}_{h}^{\mathrm{b}, \mathrm{D}} \cup \mathcal{F}_{h}^{\mathrm{b}, \mathrm{N}}$.

\subsection{Local reconstruction and stability operators}

In what follows, it is important to identify, for any mesh cell $T \in \mathcal{T}_{h}$, the part of its boundary that is not located on the subset $\Gamma_{D}$ (where Nitsche's method is employed). Thus, we set

$$
\partial T^{\backslash}:=\partial T \cap\left(\bar{\Omega} \backslash \Gamma_{D}\right)
$$

Let $\mathcal{F}_{\partial T \backslash}$ collect the faces of $T$ located on $\partial T \backslash$. Let $k \geq 0$ be the polynomial degree. For all $T \in \mathcal{T}_{h}$, the local discrete space is

$$
\widehat{U}_{T \backslash}^{k}:=\mathbb{P}^{k+1}(T) \times \mathbb{P}^{k}\left(\mathcal{F}_{\partial T}\right),
$$

that is, the local face unknowns are only attached to those faces of $T$ that are not located on $\Gamma_{D}$ (this is why we introduce the subscript $T \backslash$ rather than $T$ for the local space). A generic element in $\widehat{U}_{T \backslash}^{k}$ is denoted $\widehat{v}_{T}=\left(v_{T}, v_{\partial T \backslash}\right)$ with $v_{T} \in \mathbb{P}^{k+1}(T)$ and $v_{\partial T \backslash} \in \mathbb{P}^{k}\left(\mathcal{F}_{\partial T \backslash}\right)$.

For all $T \in \mathcal{T}_{h}$, we define the local reconstruction operator $R \backslash: \widehat{U}_{T}^{k} \rightarrow \mathbb{P}^{k+1}(T)$ such that, for all $\widehat{v}_{T}=\left(v_{T}, v_{\partial T \backslash}\right) \in \widehat{U}_{T \backslash}^{k}$,

$$
\begin{aligned}
\left(\nabla R \backslash\left(\widehat{v}_{T}\right), \nabla w\right)_{T} & =\left(\nabla v_{T}, \nabla w\right)_{T}+\left(v_{\partial T}-v_{T}, \boldsymbol{n}_{T} \cdot \nabla w\right)_{\partial T \backslash} \quad \forall w \in \mathbb{P}^{k+1}(T), \\
\left(R \backslash\left(\widehat{v}_{T}\right), 1\right)_{T} & =\left(v_{T}, 1\right)_{T},
\end{aligned}
$$

which leads, as usual, to a local well-posed Neumann problem that is solved by inverting the local stiffness matrix in $\mathbb{P}^{k+1}(T)$. The local stabilization operator $S \backslash: \widehat{U}_{T \backslash}^{k} \rightarrow \mathbb{P}^{k}\left(\mathcal{F}_{\partial T} \backslash\right)$ is defined such that, for all $\widehat{v}_{T}=\left(v_{T}, v_{\partial T \backslash}\right) \in \widehat{U}_{T \backslash}^{k}$,

$$
S \backslash\left(\widehat{v}_{T}\right):=\pi_{\partial T \backslash}^{k}\left(v_{\partial T \backslash}-v_{T \mid \partial T \backslash}\right)=v_{\partial T \backslash}-\pi_{\partial T \backslash}^{k}\left(v_{T \mid \partial T \backslash}\right) .
$$

Observe that the above form of the stabilization operator is similar (up to the restriction to $\partial T^{\backslash}$ ) to the Lehrenfeld-Schöberl stabilization in the context of mixed-order HDG methods [53].

The local bilinear form $\widehat{a}_{T}^{\backslash}$ on $\widehat{U}_{T \backslash}^{k} \times \widehat{U}_{T \backslash}^{k}$ is

$$
\widehat{a}_{T}^{\backslash}\left(\widehat{v}_{T}, \widehat{w}_{T}\right):=\left(\nabla R \backslash\left(\widehat{v}_{T}\right), \nabla R \backslash\left(\widehat{w}_{T}\right)\right)_{T}+\left(\eta_{\partial T} S^{\backslash}\left(\widehat{v}_{T}\right), S \backslash\left(\widehat{w}_{T}\right)\right)_{\partial T} \backslash
$$

where $\eta_{\partial T}$ is still the piecewise constant function on $\partial T$ (which is only needed on $\partial T^{\backslash}$ now) given by $\eta_{\partial T \mid F}=h_{F}^{-1}$ for all $F \in \mathcal{F}_{\partial T} \backslash$. We equip the discrete space $\widehat{U}_{T \backslash}^{k}$ with the following $H^{1}$-like seminorm: for all $\widehat{v}_{T}=\left(v_{T}, v_{\partial T \backslash}\right) \in \widehat{U}_{T \backslash}^{k}$

$$
\left|\widehat{v}_{T}\right|_{\widehat{U}_{T}^{k} \backslash}^{2}:=\left\|\nabla v_{T}\right\|_{T}^{2}+\left\|\eta_{\partial T}^{\frac{1}{2}}\left(v_{\partial T} \backslash-v_{T \mid \partial T} \backslash\right)\right\|_{\partial T \backslash}^{2},
$$

so that $\left|\widehat{v}_{T}\right|_{\widehat{U}_{T}^{k} \backslash}=0$ implies that $v_{T}=v_{\partial T \backslash}=c s t$. One can verify that the stability and boundedness properties from Lemma 4.1 still hold true for the discrete bilinear form $\widehat{a}_{T}$.

For all $T \in \mathcal{T}_{h}$, we define the local reduction operator $I_{T}^{\backslash}: H^{1}(T) \rightarrow \widehat{U}_{T}^{k}$ such that, for all $v \in H^{1}(T)$,

$$
I_{T}^{\backslash}(v):=\left(\pi_{T}^{k+1}(v), \pi_{\partial T \backslash}^{k}(v)\right) \in \widehat{U}_{T \backslash}^{k} .
$$


There are two differences with the usual HHO reduction operator $\widehat{I}_{T}$ considered in Lemma 4.2: for the cell component, we use a higher-order $L^{2}$-orthogonal projector onto $\mathbb{P}^{k+1}(T)$, and for the face component, we only project on those faces in $\mathcal{F}_{\partial T} \backslash$. Then,

$$
E_{T}^{\backslash}:=R \backslash I_{T}^{\backslash}: H^{1}(T) \rightarrow \mathbb{P}^{k+1}(T)
$$

still acts as an approximation operator, but it is no longer the elliptic projector, at least on those mesh cells having a boundary face in $\mathcal{F}_{h}^{\mathrm{b}, \mathrm{D}}$. It is therefore crucial at this stage to assert that $E_{T}^{\backslash}$ still enjoys optimal approximation properties. Let us recall the norm $\|v\|_{\sharp, T}^{2}:=\|\nabla v\|_{T}^{2}+h_{T}\left\|\boldsymbol{n}_{T} \cdot \nabla v\right\|_{\partial T}^{2}$, for all $v \in H^{1+s}(T)$, $s>\frac{1}{2}$, and all $T \in \mathcal{T}_{h}$.

Lemma 5.1 (Approximation). There exists a uniform constant such that the following holds true:

$$
\left\|v-E_{T}^{\backslash}(v)\right\|_{\sharp, T} \leq C\left\|v-\pi_{T}^{k+1}(v)\right\|_{\sharp, T} .
$$

for all $v \in H^{1+s}(T), s>\frac{1}{2}$, and all $T \in \mathcal{T}_{h}$. Moreover, for all $T \in \mathcal{T}_{h}$ and all $v \in H^{1}(T)$, we have

$$
\left\|\eta_{\partial T}^{\frac{1}{2}} S \backslash\left(I_{T}^{\backslash}(v)\right)\right\|_{\partial T \backslash} \leq C\left\|\nabla\left(v-\pi_{T}^{k+1}(v)\right)\right\|_{T} .
$$

Proof. To prove (5.7), let us start by bounding $\left\|\nabla\left(v-E_{T}^{\searrow}(v)\right)\right\|_{T}$. We have

$$
\begin{aligned}
\left\|\nabla\left(E_{T}^{\backslash}(v)-\pi_{T}^{k+1}(v)\right)\right\|_{T} & =\sup _{\substack{q \in \mathbb{P}^{k+1}(T) \\
\|\nabla q\|_{T}=1}}\left(\nabla\left(E_{T}^{\backslash}(v)-\pi_{T}^{k+1}(v)\right), \nabla q\right)_{T} \\
& \left.=\sup _{\substack{q \in \mathbb{P}^{k+1}(T) \\
\|\nabla q\|_{T}=1}}\left(\nabla R \backslash\left(I_{T}^{\searrow}(v)\right)-\pi_{T}^{k+1}(v)\right), \nabla q\right)_{T} \\
& =\sup _{\substack{q \in \mathbb{P}^{k+1}(T) \\
\|\nabla q\|_{T}=1}}\left(\pi_{\partial T}^{k}(v)-\pi_{T}^{k+1}(v), \boldsymbol{n}_{T} \cdot \nabla q\right)_{\partial T} \backslash \\
& =\sup _{\substack{q \in \mathbb{P}^{k+1}(T) \\
\|\nabla q\|_{T}=1}}\left(v-\pi_{T}^{k+1}(v), \boldsymbol{n}_{T} \cdot \nabla q\right)_{\partial T},
\end{aligned}
$$

where we used that $E_{T}^{\backslash}(v)-\pi_{T}^{k+1}(v) \in \mathbb{P}^{k+1}(T)$ in the first line, the definition of $E_{T}^{\searrow}$ in the second line, the definition of $R \backslash$ in the third line, and the fact that $\boldsymbol{n}_{T} \cdot \nabla q_{\mid \partial T \backslash} \in \mathbb{P}^{k}\left(\mathcal{F}_{\partial T \backslash}\right)$ in the fourth line. Using the Cauchy-Schwarz inequality followed by the discrete trace inequality from Lemma 3.1 to bound $\left\|\boldsymbol{n}_{T} \cdot \nabla q\right\|_{\partial T} \backslash$ and since $\|\nabla q\|_{T}=1$, we conclude that

$$
\left\|\nabla\left(E_{T}^{\backslash}(v)-\pi_{T}^{k+1}(v)\right)\right\|_{T} \leq C h_{T}^{-\frac{1}{2}}\left\|v-\pi_{T}^{k+1}(v)\right\|_{\partial T} .
$$

The multiplicative trace inequality from Lemma 3.2 followed by the Poincaré inequality from Lemma 3.3 then lead to

$$
\left\|\nabla\left(E_{T}^{\backslash}(v)-\pi_{T}^{k+1}(v)\right)\right\|_{T} \leq C\left\|\nabla\left(v-\pi_{T}^{k+1}(v)\right)\right\|_{T} .
$$

Let us now estimate $h_{T}^{\frac{1}{2}}\left\|\boldsymbol{n}_{T} \cdot \nabla\left(E_{T}^{\backslash}(v)-\pi_{T}^{k+1}(v)\right)\right\|_{\partial T}$. The discrete trace inequality from Lemma 3.1, estimating the normal derivative by the norm of the full gradient, and the above bound (5.9) lead to

$$
h_{T}^{\frac{1}{2}}\left\|\boldsymbol{n}_{T} \cdot \nabla\left(E_{T}^{\backslash}(v)-\pi_{T}^{k+1}(v)\right)\right\|_{\partial T} \leq C\left\|\nabla\left(E_{T}^{\backslash}(v)-\pi_{T}^{k+1}(v)\right)\right\|_{T} \leq C\left\|\nabla\left(v-\pi_{T}^{k+1}(v)\right)\right\|_{T} .
$$

We complete the proof of (5.7) by using the triangle inequality. We now turn to the proof of (5.8). Since $\pi_{\partial T \backslash}^{k} \circ \pi_{\partial T \backslash}^{k}=\pi_{\partial T \backslash}^{k}$, we have

$$
\left\|\eta_{\partial T}^{\frac{1}{2}} S^{\backslash}\left(I_{T}^{\backslash}(v)\right)\right\|_{\partial T \backslash}=\left\|\eta_{\partial T}^{\frac{1}{2}} \pi_{\partial T \backslash}^{k}\left(v-\pi_{T}^{k+1}(v)\right)\right\|_{\partial T \backslash} \leq C h_{T}^{-\frac{1}{2}}\left\|v-\pi_{T}^{k+1}(v)\right\|_{\partial T},
$$

where we used the $L^{2}$-stability of $\pi_{\partial T}^{k}$, that $\eta_{\partial T}$ is piecewise constant, and that $\partial T^{\backslash} \subseteq \partial T$. We conclude by observing that the right-hand side has been bounded above. 
Remark 5.2 (Other estimates). The bound (5.9) together with a triangle inequality implies that $\| \nabla(v-$ $\left.E_{T}^{\backslash}(v)\right)\left\|_{T} \leq C\right\| \nabla\left(v-\pi_{T}^{k+1}(v)\right) \|_{T}$. Invoking the multiplicative trace inequality from Lemma 3.2 and the Poincaré inequality from Lemma 3.3, we conclude that

$$
\left.\left\|v-E_{T}^{\backslash}(v)\right\|_{T}+h_{T}^{\frac{1}{2}} \| v-E_{T}^{\searrow}(v)\right)\left\|_{\partial T}+h_{T}\right\| \nabla\left(v-E_{T}^{\searrow}(v)\right)\left\|_{T} \leq C h_{T}\right\| \nabla\left(v-\pi_{T}^{k+1}(v)\right) \|_{T}
$$

Optimal convergence rates on $\left(v-E_{T}^{\backslash}(v)\right)$ for smooth functions $v \in H^{1+s}(T), s>\frac{1}{2}$, can then be inferred from Lemma 3.4.

\subsection{Discrete problem, stability, and well-posedness}

The definition of the global discrete space is slightly modified (we keep the same notation for simplicity) since, in the cell version, there are no face unknowns attached to those faces in $\mathcal{F}_{h}^{\mathrm{b}, \mathrm{D}}$ :

$$
\widehat{U}_{h}^{k}:=\mathbb{P}^{k+1}\left(\mathcal{T}_{h}\right) \times \mathbb{P}^{k}\left(\mathcal{F}_{h}^{\mathrm{i}} \cup \mathcal{F}_{h}^{\mathrm{b}, \mathrm{N}}\right) .
$$

A generic element in $\widehat{U}_{h}^{k}$ is denoted $\widehat{w}_{h}=\left(\left(w_{T}\right)_{T \in \mathcal{T}_{h}},\left(w_{F}\right)_{F \in \mathcal{F}_{h}^{\mathrm{i}} \cup \mathcal{F}_{h}^{\mathrm{b}, \mathrm{N}}}\right)$, and for all $T \in \mathcal{T}_{h}$, we denote by

$$
\widehat{w}_{T}=\left(w_{T},\left(w_{F}\right)_{F \in \mathcal{F}_{\partial T} \backslash}\right) \in \widehat{U}_{T \backslash}^{k}
$$

the components of $\widehat{w}_{h}$ attached to the mesh cell $T$ and its faces composing $\partial T$. We consider the following discrete Nitsche-HHO problem:

$$
\left\{\begin{array}{l}
\text { Find } \widehat{u}_{h} \in \widehat{U}_{h}^{k} \text { such that } \\
\widehat{a}_{h}\left(\widehat{u}_{h}, \widehat{w}_{h}\right)=\widehat{\ell}_{h}\left(\widehat{w}_{h}\right) \quad \forall \widehat{w}_{h} \in \widehat{U}_{h}^{k} .
\end{array}\right.
$$

For all $\widehat{v}_{h}, \widehat{w}_{h} \in \widehat{U}_{h}^{k}$, the global discrete bilinear form $\widehat{a}_{h}$ and the global discrete linear form $\widehat{\ell}_{h}$ are defined respectively by (compare with (4.9))

$$
\begin{aligned}
\widehat{a}_{h}\left(\widehat{v}_{h}, \widehat{w}_{h}\right):= & \sum_{T \in \mathcal{T}_{h}} \widehat{a}_{T}\left(\widehat{v}_{T}, \widehat{w}_{T}\right)-\sum_{F \in \mathcal{F}_{h}^{b, D}}\left(\sigma_{n}\left(R \backslash\left(\widehat{v}_{T(F)}\right)\right), w_{T(F)}\right)_{F} \\
& -\sum_{F \in \mathcal{F}_{h}^{b, D}} \theta\left(v_{T(F)}, \sigma_{n}\left(R \backslash\left(\widehat{w}_{T(F)}\right)\right)\right)_{F}+\sum_{F \in \mathcal{F}_{h}^{b, D}} \gamma\left(v_{T(F)}, w_{T(F)}\right)_{F}, \\
\widehat{\ell}_{h}\left(\widehat{w}_{h}\right):= & \sum_{T \in \mathcal{T}_{h}}\left(f, w_{T}\right)_{T}+\sum_{F \in \mathcal{F}_{h}^{b, N}}\left(g_{N}, w_{F}\right)_{F} \\
& -\sum_{F \in \mathcal{F}_{h}^{b, D}} \theta\left(g_{D}, \sigma_{n}\left(R \backslash\left(\widehat{w}_{T(F)}\right)\right)\right)_{F}+\sum_{F \in \mathcal{F}_{h}^{b, D}} \gamma\left(g_{D}, w_{T(F)}\right)_{F} .
\end{aligned}
$$

Comparing (4.9a) with (5.11a), we see that $v_{T(F)}$ and $w_{T(F)}$ are now used in place of $v_{F}$ and $w_{F}$ in the three terms on $\mathcal{F}_{h}^{\mathrm{b}, \mathrm{D}}$ defining $\widehat{a}_{h}$, and comparing $(4.9 \mathrm{~b})$ with $(5.11 \mathrm{~b})$, we see that $w_{T(F)}$ is now used in place of $w_{F}$ in the penalty term on $\mathcal{F}_{h}^{\mathrm{b}, \mathrm{D}}$ defining $\widehat{\ell}_{h}$. Notice, however, that the enforcement of the Neumann condition in $\widehat{\ell}_{h}$ still involves the face component $w_{F}$ of the test function $\widehat{w}_{h}$.

We equip the space $\widehat{U}_{h}^{k}$ with the norm

$$
\left\|\widehat{v}_{h}\right\|_{\widehat{U}_{h}^{k}}^{2}:=\sum_{T \in \mathcal{T}_{h}}\left|\widehat{v}_{T}\right|_{\widehat{U}_{T}^{k} \backslash}^{2}+\sum_{F \in \mathcal{F}_{h}^{\mathrm{b}, \mathrm{D}}} h_{F}^{-1}\left\|v_{T(F)}\right\|_{F}^{2}, \quad \forall \widehat{v}_{h} \in \widehat{U}_{h}^{k},
$$

with the local seminorms $|\cdot|_{\widehat{U}_{T}^{k} \backslash}$ defined in (5.5). That $\|\cdot\|_{\widehat{U}_{h}^{k}}$ defines a norm on $\widehat{U}_{h}^{k}$ follows from similar arguments to those considered for the face version in the previous section. Let us now address the wellposedness of the discrete problem (5.10). As above, we only consider $\theta=1$. Well-posedness also holds true for $\theta=0$ (with a less stringent lower bound on $\gamma_{0}$ ) and for $\theta=-1$ (with the simple requirement that $\left.\gamma_{0}>0\right)$. 
Lemma 5.3 (Coercivity and well-posedness). Assume that $\theta=1$ and that $\gamma_{0}>2 n^{\mathrm{b}, \mathrm{D}} C_{\mathrm{dt}}^{2}$, where $C_{\mathrm{dt}}$ results from the discrete trace inequality of Lemma 3.1. Let us set the penalty parameter to $\gamma_{\mid F}:=\gamma_{0} h_{F}^{-1}$, for all $F \in \mathcal{F}_{h}^{\mathrm{b}, \mathrm{D}}$. Then the discrete bilinear form $\widehat{a}_{h}$ is coercive, and the discrete problem (5.10) is well-posed.

Proof. Identical to the proof of Lemma 4.4 .

\subsection{Error analysis}

We carry out the error analysis for $\theta=1$; the proofs for the other values $\theta \in\{-1,0\}$ follow by minor adaptations of the arguments for $\theta=1$. As before, the first important step in the error analysis is to bound the consistency error; we recall that this error is defined by (4.11), where the global reduction operator $\widehat{I}_{h}: H^{1}(\Omega) \rightarrow \widehat{U}_{h}^{k}$ is now such that the local components of $\widehat{I}_{h}(v)$, for all $v \in H^{1}(\Omega)$, attached to a mesh cell $T \in \mathcal{T}_{h}$, are $I_{T}^{\backslash}\left(v_{\mid T}\right)$.

Lemma 5.4 (Consistency). Assume that $\theta=1$ and $u \in H^{1+s}(\Omega)$ with $s>\frac{1}{2}$. The following holds true:

$$
\left|\mathcal{E}_{h}\left(\widehat{w}_{h}\right)\right| \lesssim\left(\sum_{T \in \mathcal{T}_{h}}\left\|u-\pi_{T}^{k+1}(u)\right\|_{\sharp, T}^{2}\right)^{\frac{1}{2}}\left\|\widehat{w}_{h}\right\|_{\widehat{U}_{h}^{k}}, \quad \forall \widehat{w}_{h} \in \widehat{U}_{h}^{k} .
$$

Proof. Let $\widehat{w}_{h} \in \widehat{U}_{h}^{k}$. Let us introduce the shorthand notation $\eta_{\gamma}\left(\widehat{w}_{T(F)}\right):=-\sigma_{n}\left(R \backslash\left(\widehat{w}_{T(F)}\right)\right)+\gamma w_{T(F)}$. Using the definitions (5.11a)-(5.11b) of $\widehat{a}_{h}, \widehat{\ell}_{h}$, the PDE and the boundary conditions satisfied by the exact solution $u$, and since $R \backslash I_{T}^{\backslash}=E_{T}^{\backslash}$, we have

$$
\begin{aligned}
\mathcal{E}_{h}\left(\widehat{w}_{h}\right)= & \sum_{T \in \mathcal{T}_{h}}\left(-\Delta u, w_{T}\right)_{T}+\sum_{F \in \mathcal{F}_{h}^{b, N}}\left(\sigma_{n}(u), w_{F}\right)_{F}+\sum_{F \in \mathcal{F}_{h}^{b, D}}\left(u, \eta_{\gamma}\left(\widehat{w}_{T(F)}\right)\right)_{F} \\
& -\sum_{T \in \mathcal{T}_{h}}\left(\nabla E_{T}^{\backslash}(u), \nabla R \backslash\left(\widehat{w}_{T}\right)\right)_{T}-\sum_{T \in \mathcal{T}_{h}}\left(\eta_{\partial T} S^{\backslash}\left(I_{T}^{\backslash}(u)\right), S^{\backslash}\left(\widehat{w}_{T}\right)\right)_{\partial T} \backslash \\
& +\sum_{F \in \mathcal{F}_{h}^{b, D}}\left(\sigma_{n}\left(E_{T(F)}^{\backslash}(u)\right), w_{T(F)}\right)_{F}-\left(\pi_{T(F)}^{k+1}(u), \eta_{\gamma}\left(\widehat{w}_{T(F)}\right)\right)_{F} .
\end{aligned}
$$

Re-arranging terms leads to

$$
\mathcal{E}_{h}\left(\widehat{w}_{h}\right)=\mathcal{T}_{1}-\mathcal{T}_{2}-\mathcal{T}_{3}+\mathcal{T}_{4}
$$

where

$$
\begin{aligned}
& \mathcal{T}_{1}=\sum_{T \in \mathcal{T}_{h}}\left(-\Delta u, w_{T}\right)_{T}+\sum_{F \in \mathcal{F}_{h}^{b, N}}\left(\sigma_{n}(u), w_{F}\right)_{F}, \\
& \mathcal{T}_{2}=\sum_{T \in \mathcal{T}_{h}}\left(\nabla E_{T}^{\backslash}(u), \nabla R^{\backslash}\left(\widehat{w}_{T}\right)\right)_{T}-\sum_{F \in \mathcal{F}_{h}^{b, D}}\left(\sigma_{n}\left(E_{T(F)}^{\backslash}(u)\right), w_{T(F)}\right)_{F}, \\
& \mathcal{T}_{3}=\sum_{T \in \mathcal{T}_{h}}\left(\eta_{\partial T} S^{\backslash}\left(I_{T}^{\backslash}(u)\right), S \backslash\left(\widehat{w}_{T}\right)\right)_{\partial T} \backslash \\
& \mathcal{T}_{4}=\sum_{F \in \mathcal{F}_{h}^{b, D}}\left(u-\pi_{T(F)}^{k+1}(u), \eta_{\gamma}\left(\widehat{w}_{T(F)}\right)\right)_{F} .
\end{aligned}
$$

Note that a term of the form $\mathcal{T}_{4}$ is not present in the consistency error of the face version of Nitsche-HHO. Integrating by parts in each mesh cell and using that $\sigma_{n}(u)$ is single-valued across all the mesh interfaces (and well-defined since $s>\frac{1}{2}$ ), we obtain

$$
\begin{aligned}
\mathcal{T}_{1} & =\sum_{T \in \mathcal{T}_{h}}\left(\nabla u, \nabla w_{T}\right)_{T}-\sum_{T \in \mathcal{T}_{h}}\left(\sigma_{n}(u), w_{T}\right)_{\partial T}+\sum_{F \in \mathcal{F}_{h}^{b, N}}\left(\sigma_{n}(u), w_{F}\right)_{F} \\
& =\sum_{T \in \mathcal{T}_{h}}\left(\nabla u, \nabla w_{T}\right)_{T}-\sum_{T \in \mathcal{T}_{h}}\left(\sigma_{n}(u), w_{T}-w_{\partial T \backslash}\right)_{\partial T \backslash}-\sum_{F \in \mathcal{F}_{h}^{b, D}}\left(\sigma_{n}(u), w_{T(F)}\right)_{F} .
\end{aligned}
$$


Comparing with the term $\mathcal{T}_{1}$ from the consistency proof of the face version, we see that on the right-hand side, the second term is now restricted to $\partial T^{\backslash}$ and that the third term is now evaluated using $w_{T(F)}$ instead of $w_{F}$. Moreover, using the definition of $R \backslash\left(\widehat{w}_{T}\right)$, we infer that

$$
\mathcal{T}_{2}=\sum_{T \in \mathcal{T}_{h}}\left(\nabla E_{T}^{\backslash}(u), \nabla w_{T}\right)_{T}-\sum_{T \in \mathcal{T}_{h}}\left(\sigma_{n}\left(E_{T}^{\backslash}(u)\right), w_{T}-w_{\partial T \backslash}\right)_{\partial T \backslash}-\sum_{F \in \mathcal{F}_{h}^{b, D}}\left(\sigma_{n}\left(E_{T(F)}^{\backslash}(u)\right), w_{T(F)}\right)_{F} .
$$

Consequently, if we define for all $T \in \mathcal{T}_{h}$ the function $\delta_{T}:=u_{\mid T}-E_{T}^{\backslash}\left(u_{\mid T}\right)$, we obtain

$$
\mathcal{T}_{1}-\mathcal{T}_{2}=\sum_{T \in \mathcal{T}_{h}}\left(\nabla \delta_{T}, \nabla w_{T}\right)_{T}-\sum_{T \in \mathcal{T}_{h}}\left(\sigma_{n}\left(\delta_{T}\right), w_{T}-w_{\partial T} \backslash\right)_{\partial T} \backslash-\sum_{F \in \mathcal{F}_{h}^{b, D}}\left(\sigma_{n}\left(\delta_{T(F)}\right), w_{T(F)}\right)_{F} .
$$

We can now bound $\mathcal{T}_{1}-\mathcal{T}_{2}$ by proceeding as in the analysis of the face version (note though that the first term on the above right-hand side does no longer vanish). Moreover, the bound on $\mathcal{T}_{3}$ is identical to that for the face version. Finally, we bound $\mathcal{T}_{4}$ by means of the Cauchy-Schwarz inequality and observing that $h_{T(F)}^{-\frac{1}{2}}\left\|u-\pi_{T(F)}^{k+1}(u)\right\|_{F} \lesssim\left\|\nabla\left(u-\pi_{T(F)}^{k+1}(u)\right)\right\|_{T(F)}$, as already argued in the proof of Lemma 5.1 , and that

$$
\begin{aligned}
h_{T(F)}^{\frac{1}{2}}\left\|\eta_{\gamma}\left(\widehat{w}_{T(F)}\right)\right\|_{F} & \leq h_{T(F)}^{\frac{1}{2}}\left\|\sigma_{n}\left(R \backslash\left(\widehat{w}_{T(F)}\right)\right)\right\|_{F}+\gamma_{0} h_{T(F)}^{-\frac{1}{2}}\left\|w_{T(F)}\right\|_{F} \\
& \lesssim\left\|\nabla R \backslash\left(\widehat{w}_{T(F)}\right)\right\|_{T(F)}+h_{T(F)}^{-\frac{1}{2}}\left\|w_{T(F)}\right\|\left\|_{F} \lesssim\left|\widehat{w}_{T}\right|_{\widehat{U}_{T \backslash}^{k}}+h_{T(F)}^{-\frac{1}{2}}\right\| w_{T(F)} \|_{F},
\end{aligned}
$$

owing to the triangle inequality, the discrete trace inequality from Lemma 3.1, and the boundedness of the local bilinear form $\widehat{a}_{T}$ defined in (5.4).

Theorem 5.5 ( $H^{1}$-error estimate). Assume that $u \in H^{1+s}(\Omega)$ with $s>\frac{1}{2}$. The following holds true:

$$
\sum_{T \in \mathcal{T}_{h}}\left\|\nabla\left(u-R \backslash\left(\widehat{u}_{T}\right)\right)\right\|_{T}^{2} \lesssim \sum_{T \in \mathcal{T}_{h}}\left\|u-\pi_{T}^{k+1}(u)\right\|_{\sharp, T}^{2}
$$

Consequently, letting $t:=\min (k+1, s)$, we have

$$
\sum_{T \in \mathcal{T}_{h}}\left\|\nabla\left(u-R \backslash\left(\widehat{u}_{T}\right)\right)\right\|_{T}^{2} \lesssim \sum_{T \in \mathcal{T}_{h}} h_{T}^{2 t}|u|_{H^{2+t}(T)}^{2}
$$

Proof. The proof of (5.14) uses Lemma 5.4 and proceeds as that of (4.13) for the face version. Finally, the estimate (5.15) is a consequence of (5.14) and Lemma 3.4.

\section{Signorini conditions}

In this section, we devise and analyze a Nitsche-HHO method to approximate the Signorini conditions in the model problem (2.4)-(2.5). We consider the cell version, still with a mixed-order, where the cell unknowns are of one order higher than the face unknowns. We assume that the meshes are compatible with the boundary partition $\partial \Omega=\overline{\Gamma_{D}} \cup \overline{\Gamma_{N}} \cup \overline{\Gamma_{S}}$ from (2.2), which leads to the partition of boundary faces as $\mathcal{F}_{h}^{\mathrm{b}}=\mathcal{F}_{h}^{\mathrm{b}, \mathrm{D}} \cup \mathcal{F}_{h}^{\mathrm{b}, \mathrm{N}} \cup \mathcal{F}_{h}^{\mathrm{b}, \mathrm{S}}$ (with obvious notation). For simplicity, we employ the Nitsche technique only on the subset $\Gamma_{S}$ where the nonlinear Signorini conditions are enforced, whereas we resort to a strong enforcement of the homogeneous Dirichlet condition on the subset $\Gamma_{D}$. Note however that the numerical analysis of this section can be readily extended to the case where Nitsche's method is used also for the Dirichlet boundary condition.

\subsection{Local reconstruction and stability operators}

For simplicity, we keep the same notation as in the previous section, although we keep in mind that we are now concerned with the subset $\Gamma_{S}$ rather than $\Gamma_{D}$. For all $T \in \mathcal{T}_{h}$, we identify the part of its boundary that is not located on the subset $\Gamma_{S}$ (where Nitsche's method is employed):

$$
\partial T^{\backslash}:=\partial T \cap\left(\bar{\Omega} \backslash \Gamma_{S}\right)
$$


and we let, as before, $\mathcal{F}_{\partial T \backslash} \backslash$ collect the faces of $T$ located on $\partial T \backslash$. Let $k \geq 0$ be the polynomial degree. For all $T \in \mathcal{T}_{h}$, the local discrete space is:

$$
\widehat{U}_{T \backslash}^{k}:=\mathbb{P}^{k+1}(T) \times \mathbb{P}^{k}\left(\mathcal{F}_{\partial T \backslash}\right),
$$

that is, the local face unknowns are only attached to those faces of $T$ that are not located in $\Gamma_{S}$. A generic element in $\widehat{U}_{T \backslash}^{k}$ is denoted $\widehat{v}_{T}=\left(v_{T}, v_{\partial T}\right)$ with $v_{T} \in \mathbb{P}^{k+1}(T)$ and $v_{\partial T \backslash} \in \mathbb{P}^{k}\left(\mathcal{F}_{\partial T} \backslash\right)$.

For all $T \in \mathcal{T}_{h}$, the local reconstruction operator $R \backslash: \widehat{U}_{T \backslash}^{k} \rightarrow \mathbb{P}^{k+1}(T)$ is still defined by (5.1), and the local stabilization operator $S^{\backslash}: \widehat{U}_{T \backslash}^{k} \rightarrow \mathbb{P}^{k}\left(\mathcal{F}_{\partial T} \backslash\right)$ is still defined by (5.3). The local bilinear form $\widehat{a}_{T}^{\backslash}$ on $\widehat{U}_{T \backslash}^{k} \times \widehat{U}_{T \backslash}^{k}$ is still defined by (5.4). We equip the discrete space $\widehat{U}_{T \backslash}^{k}$ with the $H^{1}$-like seminorm $|\cdot|_{U_{T}^{k}}$ defined by (5.5), and we recall that the discrete bilinear form $\widehat{a}_{T}$ satisfies the stability and boundedness properties from Lemma 4.1. We let the local reduction operator $I_{T}^{\backslash}: H^{1}(T) \rightarrow \widehat{U}_{T \backslash}^{k}$ be defined by (5.6), and, as before, we let $E_{T}^{\backslash}:=R \backslash \circ I_{T}^{\backslash}: H^{1}(T) \rightarrow \mathbb{P}^{k+1}(T)$. The approximation properties of $E_{T}^{\backslash}$ and of $S \backslash \circ I_{T}^{\backslash}$ are those stated in Lemma 5.1.

\subsection{Discrete problem and well-posedness}

The global discrete space is

$$
\widehat{U}_{h}^{k}:=\mathbb{P}^{k+1}\left(\mathcal{T}_{h}\right) \times \mathbb{P}^{k}\left(\mathcal{F}_{h}^{\mathrm{i}} \cup \mathcal{F}_{h}^{\mathrm{b}, \mathrm{D}} \cup \mathcal{F}_{h}^{\mathrm{b}, \mathrm{N}}\right) .
$$

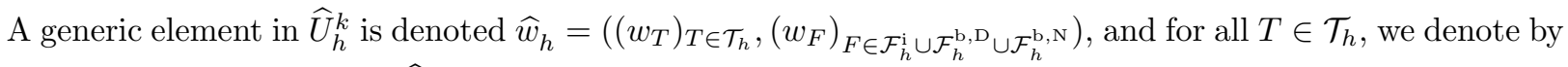
$\widehat{w}_{T}=\left(w_{T},\left(w_{F}\right)_{F \in \mathcal{F}_{\partial T} \backslash}\right) \in \widehat{U}_{T \backslash}^{k}$ the components of $\widehat{w}_{h}$ attached to the mesh cell $T$ and its faces composing $\partial T \backslash$. We enforce strongly the homogeneous Dirichlet condition on $\Gamma_{D}$ by considering the subspace

$$
\widehat{U}_{h, 0}^{k}:=\left\{\widehat{w}_{h} \in \widehat{U}_{h}^{k} \mid w_{F}=0 \quad \forall F \in \mathcal{F}_{h}^{\mathrm{b}, \mathrm{D}}\right\} .
$$

We consider the following discrete Nitsche-HHO problem:

$$
\left\{\begin{array}{l}
\text { Find } \widehat{u}_{h} \in \widehat{U}_{h, 0}^{k} \text { such that } \\
\widehat{a}_{h}\left(\widehat{u}_{h} ; \widehat{w}_{h}\right)=\widehat{\ell}\left(\widehat{w}_{h}\right) \quad \forall \widehat{w}_{h} \in \widehat{U}_{h, 0}^{k} .
\end{array}\right.
$$

For all $\widehat{v}_{h}, \widehat{w}_{h} \in \widehat{U}_{h, 0}^{k}$, the global discrete semilinear form $\widehat{a}_{h}$ and the global discrete linear form $\widehat{\ell}$ are defined respectively by (compare with $(2.7)$ )

$$
\begin{aligned}
\widehat{a}_{h}\left(\widehat{v}_{h} ; \widehat{w}_{h}\right):= & \sum_{T \in \mathcal{T}_{h}} \widehat{a}_{T}\left(\widehat{v}_{T}, \widehat{w}_{T}\right)-\sum_{F \in \mathcal{F}_{h}^{b, S}} \frac{\theta}{\gamma}\left(\sigma_{n}\left(R \backslash\left(\widehat{v}_{T(F)}\right)\right), \sigma_{n}\left(R \backslash\left(\widehat{w}_{T(F)}\right)\right)\right)_{F} \\
& +\sum_{F \in \mathcal{F}_{h}^{b, S}} \frac{1}{\gamma}\left(\left[\widehat{\phi}_{\gamma}\left(\widehat{v}_{T(F)}\right)\right]_{\mathbb{R}^{-}}, \widehat{\phi}_{\gamma \theta}\left(\widehat{w}_{T(F)}\right)\right)_{F}, \\
\widehat{\ell}\left(\widehat{w}_{h}\right):= & \sum_{T \in \mathcal{T}_{h}}\left(f, w_{T}\right)_{T}+\sum_{F \in \mathcal{F}_{h}^{b, N}}\left(g_{N}, w_{F}\right)_{F},
\end{aligned}
$$

where

$$
\begin{aligned}
\widehat{\phi}_{\gamma \theta}\left(\widehat{w}_{T(F)}\right) & :=\theta \sigma_{n}\left(R \backslash\left(\widehat{w}_{T(F)}\right)\right)-\gamma w_{T(F)}, \\
\widehat{\phi}_{\gamma}\left(\widehat{w}_{T(F)}\right) & :=\widehat{\phi}_{\gamma 1}\left(\widehat{w}_{T(F)}\right)=\sigma_{n}\left(R \backslash\left(\widehat{w}_{T(F)}\right)\right)-\gamma w_{T(F)} .
\end{aligned}
$$

Note that we are employing the trace of the cell unknown in the definition of $\widehat{\phi}_{\gamma \theta}$ and $\widehat{\phi}_{\gamma}$.

We equip the space $\widehat{U}_{h, 0}^{k}$ with the norm

$$
\left\|\widehat{v}_{h}\right\|_{\widehat{U}_{h, 0}^{k}}^{2}:=\sum_{T \in \mathcal{T}_{h}}\left|\widehat{v}_{T}\right|_{\widehat{U}_{T \backslash}^{k}}^{2}, \quad \forall \widehat{v}_{h} \in \widehat{U}_{h, 0}^{k}
$$


with the local seminorms $|\cdot|_{\widehat{U}_{T}^{k}}$ defined in (5.5). That $\|\cdot\|_{\widehat{U}_{h, 0}^{k}}$ defines a norm on $\widehat{U}_{h, 0}^{k}$ follows from the usual arguments, keeping in mind that the subset $\mathcal{F}_{h}^{\mathrm{b}, \mathrm{D}}$, where the face unknowns are set to zero, is nonempty. Let us now address the well-posedness of the discrete problem (6.1). As above, we only consider $\theta=1$. Well-posedness also holds true for $\theta=0$ (with a less stringent lower bound on $\gamma_{0}$ ) and for $\theta=-1$ (with the simple requirement that $\gamma_{0}>0$ ). Let $n^{\mathrm{b}, \mathrm{S}}$ be the maximum number of faces in $\mathcal{F}_{h}^{\mathrm{b}, \mathrm{S}}$ that a mesh cell can have $\left(n^{\mathrm{b}, \mathrm{S}} \leq d\right.$ on simplicial meshes). In what follows, we use the fact that

$$
\left([x]_{\mathbb{R}^{-}}-[y]_{\mathbb{R}^{-}}\right)(x-y) \geq\left([x]_{\mathbb{R}^{-}}-[y]_{\mathbb{R}^{-}}\right)^{2} \geq 0, \quad \forall x, y \in \mathbb{R} .
$$

Lemma 6.1 (Well-posedness). Assume that $\theta=1$ and that $\gamma_{0} \geq 2 n^{\mathrm{b}, \mathrm{S}} C_{\mathrm{dt}}^{2}$, where $C_{\mathrm{dt}}$ results from the discrete trace inequality of Lemma 3.1. Let us set the penalty parameter to $\gamma_{\mid F}:=\gamma_{0} h_{F}^{-1}$, for all $F \in \mathcal{F}_{h}^{\mathrm{b}, \mathrm{S}}$. Then the discrete problem (6.1) is well-posed.

Proof. Let us first prove the following monotonicity property: for $\gamma_{0} \geq 2 n^{\mathrm{b}, \mathrm{S}} C_{\mathrm{dt}}^{2}$, there is $\alpha>0$, uniform with respect to $h$, such that, for all $\widehat{v}_{h}, \widehat{w}_{h} \in \widehat{U}_{h, 0}^{k}$,

$$
\widehat{a}_{h}\left(\widehat{v}_{h} ; \widehat{v}_{h}-\widehat{w}_{h}\right)-\widehat{a}_{h}\left(\widehat{w}_{h} ; \widehat{v}_{h}-\widehat{w}_{h}\right) \geq \alpha\left\|\widehat{v}_{h}-\widehat{w}_{h}\right\|_{\widehat{U}_{h, 0}^{k}}^{2}+\Delta \widehat{\phi}_{\gamma}\left(\widehat{v}_{h}, \widehat{w}_{h}\right),
$$

with the shorthand notation

$$
\Delta \widehat{\phi}_{\gamma}\left(\widehat{v}_{h}, \widehat{w}_{h}\right):=\sum_{F \in \mathcal{F}_{h}^{\mathrm{b}, \mathrm{S}}} \frac{1}{\gamma}\left(\left[\widehat{\phi}_{\gamma}\left(\widehat{v}_{T(F)}\right)\right]_{\mathbb{R}^{-}}-\left[\widehat{\phi}_{\gamma}\left(\widehat{w}_{T(F)}\right)\right]_{\mathbb{R}^{-}}, \widehat{\phi}_{\gamma}\left(\widehat{v}_{T(F)}\right)-\widehat{\phi}_{\gamma}\left(\widehat{w}_{T(F)}\right)\right)_{F} .
$$

Note that the identity (6.5) implies that $\Delta \widehat{\phi}_{\gamma}\left(\widehat{v}_{h}, \widehat{w}_{h}\right) \geq 0$. Moreover, we have

$$
\begin{aligned}
& \widehat{a}_{h}\left(\widehat{v}_{h} ; \widehat{v}_{h}-\widehat{w}_{h}\right)-\widehat{a}_{h}\left(\widehat{w}_{h} ; \widehat{v}_{h}-\widehat{w}_{h}\right) \\
& =\sum_{T \in \mathcal{T}_{h}} \widehat{a}_{T}^{\backslash}\left(\widehat{v}_{T}-\widehat{w}_{T}, \widehat{v}_{T}-\widehat{w}_{T}\right)-\sum_{F \in \mathcal{F}_{h}^{\mathrm{b}, \mathrm{S}}} \frac{h_{F}}{\gamma_{0}}\left\|\sigma_{n}\left(R \backslash\left(\widehat{v}_{T(F)}-\widehat{w}_{T(F)}\right)\right)\right\|_{F}^{2}+\Delta \widehat{\phi}_{\gamma}\left(\widehat{v}_{h}, \widehat{w}_{h}\right) .
\end{aligned}
$$

Let us denote by $\mathcal{T}_{1}$ and $\mathcal{T}_{2}$ the first two terms on the above right-hand side. We use the lower bound on $\gamma_{0}$ and the discrete trace inequality from Lemma 3.1 to infer that

$$
\mathcal{T}_{1}+\mathcal{T}_{2} \geq \frac{1}{2} \sum_{T \in \mathcal{T}_{h}} \widehat{a}_{T}^{\backslash}\left(\widehat{v}_{T}-\widehat{w}_{T}, \widehat{v}_{T}-\widehat{w}_{T}\right)
$$

and the stability property of $\widehat{a}_{T}^{\backslash}$ in the seminorm $|\cdot|_{\widehat{U}_{T}^{k} \backslash}$ then implies that $\mathcal{T}_{1}+\mathcal{T}_{2} \geq \alpha\left\|\widehat{v}_{h}-\widehat{w}_{h}\right\|_{\widehat{U}_{h, 0}^{k}}^{2}$ for some uniform positive constant $\alpha$. This proves the monotonicity property (6.6). To infer well-posedness from this property, we use the argument from $\left[10\right.$, Corollary 15, p. 126]. Let $(\cdot, \cdot)_{\widehat{U}_{h, 0}^{k}}$ denote the inner product associated with the norm $\|\cdot\|_{\widehat{U}_{h, 0}^{k}}$. We define the nonlinear operator $B_{h}: \widehat{U}_{h, 0}^{k} \rightarrow \widehat{U}_{h, 0}^{k}$ so that $\left(B_{h}\left(\widehat{v}_{h}\right), \widehat{w}_{h}\right)_{\widehat{U}_{h, 0}^{k}}=\widehat{a}_{\gamma 1, h}\left(\widehat{v}_{h} ; \widehat{w}_{h}\right)$, for all $\widehat{v}_{h}, \widehat{w}_{h} \in \widehat{U}_{h, 0}^{k}$. We prove as in [19] that $B_{h}$ is hemicontinuous and, invoking (6.6), we conclude that $B_{h}$ is a one-to-one operator.

Remark 6.2. For $\theta=1$, the Nitsche-HHO formulation (6.1) can be recovered as the first-order optimality condition of the functional

$$
\begin{aligned}
\mathcal{J}_{h}^{S}\left(\widehat{v}_{h}\right):= & \frac{1}{2} \sum_{T \in \mathcal{T}_{h}} \widehat{a}_{T}\left(\widehat{v}_{T}, \widehat{v}_{T}\right)-\sum_{F \in \mathcal{F}_{h}^{b, S}} \frac{1}{2 \gamma}\left\|\sigma_{n}\left(R \backslash\left(\widehat{v}_{T(F)}\right)\right)\right\|_{F}^{2} \\
& +\sum_{F \in \mathcal{F}_{h}^{b, S}} \frac{1}{2 \gamma}\left\|\left[\widehat{\phi}_{\gamma}\left(\widehat{v}_{T(F)}\right)\right]_{\mathbb{R}^{-}}\right\|_{F}^{2}-\sum_{T \in \mathcal{T}_{h}}\left(f, w_{T}\right)_{T}-\sum_{F \in \mathcal{F}_{h}^{b, N}}\left(g_{N}, w_{F}\right)_{F},
\end{aligned}
$$

for all $\widehat{v}_{h} \in \widehat{U}_{h, 0}^{k}$. Lemma 6.1 implies that for $\gamma_{0}$ large enough, $\mathcal{J}_{h}^{S}$ is strongly convex. 


\subsection{Error analysis}

The consistency error is defined by

$$
\mathcal{E}_{h}\left(\widehat{w}_{h}\right):=\widehat{\ell}\left(\widehat{w}_{h}\right)-\widehat{a}_{h}\left(\widehat{I}_{h}(u) ; \widehat{w}_{h}\right), \quad \forall \widehat{w}_{h} \in \widehat{U}_{h, 0}^{k},
$$

with the global reduction operator $\widehat{I}_{h}: H_{D}^{1}(\Omega):=\left\{v \in H^{1}(\Omega) \mid v_{\mid \Gamma_{D}}=0\right\} \rightarrow \widehat{U}_{h, 0}^{k}$ such that the local components of $\widehat{I}_{h}(v)$, for all $v \in H_{D}^{1}(\Omega)$, attached to a mesh cell $T \in \mathcal{T}_{h}$, are $I_{T}^{\backslash}\left(v_{\mid T}\right)$. Owing to the nonlinearity of $\widehat{a}_{h}$ in its first argument, we will not proceed with the same level of generality as in the linear case by bounding the consistency error acting on an arbitrary test function $\widehat{w}_{h} \in \widehat{U}_{h, 0}^{k}$. We will consider more specifically the test function

$$
\widehat{z}_{h}:=\widehat{u}_{h}-\widehat{I}_{h}(u) \in \widehat{U}_{h, 0}^{k} .
$$

We only give proofs for $\theta=1$; the proofs for $\theta \in\{-1,0\}$ follow by minor adaptations of the arguments for $\theta=1$. Recall that $\|v\|_{\sharp, T}^{2}:=\|\nabla v\|_{T}^{2}+h_{T}\left\|\boldsymbol{n}_{T} \cdot \nabla v\right\|_{\partial T}^{2}$ for any function $v \in H^{1+s}(T), s>\frac{1}{2}$.

Lemma 6.3 (Consistency). Assume that $u \in H^{1+s}(\Omega)$ with $s>\frac{1}{2}$. Let $\widehat{z}_{h}:=\widehat{u}_{h}-\widehat{I}_{h}(u)$. There is a uniform constant $C$ such that the following holds true:

$$
\begin{aligned}
& \mathcal{E}_{h}\left(\widehat{z}_{h}\right)+\sum_{F \in \mathcal{F}_{h}^{\mathrm{b}, \mathrm{S}}} \frac{1}{2 \gamma}\left\|\left[\phi_{\gamma}(u)\right]_{\mathbb{R}^{-}}-\left[\widehat{\phi}_{\gamma}\left(\widehat{u}_{T(F)}\right)\right]_{\mathbb{R}^{-}}\right\|_{F}^{2} \\
& \lesssim\left(\sum_{T \in \mathcal{T}_{h}}\left\|u-\pi_{T}^{k+1}(u)\right\|_{\sharp, T}^{2}\right)^{\frac{1}{2}}\left\|\widehat{z}_{h}\right\|_{\widehat{U}_{h, 0}^{k}}+\sum_{T \in \mathcal{T}_{h}}\left\|u-\pi_{T}^{k+1}(u)\right\|_{\sharp, T}^{2}+\Delta \widehat{\phi}_{\gamma}\left(\widehat{u}_{h}, \widehat{I}_{h}(u)\right),
\end{aligned}
$$

with the notation $\Delta \widehat{\phi}_{\gamma}\left(\widehat{v}_{h}, \widehat{w}_{h}\right)$ defined in (6.7).

Proof. Using the PDE and the Neumann boundary condition satisfied by the exact solution $u$, and since $R \backslash I_{T}^{\backslash}=E_{T}^{\backslash}$ for all $T \in \mathcal{T}_{h}$, we have

$$
\begin{aligned}
\mathcal{E}_{h}\left(\widehat{z}_{h}\right)= & \sum_{T \in \mathcal{T}_{h}}\left(-\Delta u, z_{T}\right)_{T}+\sum_{F \in \mathcal{F}_{h}^{\mathrm{b}, \mathrm{N}}}\left(\sigma_{n}(u), z_{F}\right)_{F} \\
& -\sum_{T \in \mathcal{T}_{h}} \widehat{a}_{T}\left(I_{T}^{\backslash}(u), \widehat{z}_{T}\right)+\sum_{F \in \mathcal{F}_{h}^{\mathrm{b}, \mathrm{S}}} \frac{1}{\gamma}\left(\sigma_{n}\left(E_{T(F)}^{\backslash}(u)\right), \sigma_{n}\left(R \backslash\left(\widehat{z}_{T(F)}\right)\right)\right)_{F} \\
& -\sum_{F \in \mathcal{F}_{h}^{\mathrm{b}, \mathrm{S}}} \frac{1}{\gamma}\left(\left[\widehat{\phi}_{\gamma}\left(I_{T(F)}^{\backslash}(u)\right)\right]_{\mathbb{R}^{-}}, \widehat{\phi}_{\gamma}\left(\widehat{z}_{T(F)}\right)\right)_{F} \cdot
\end{aligned}
$$

Adding and subtracting $\sum_{F \in \mathcal{F}_{h}^{\mathrm{b}, \mathrm{S}}}\left(\sigma_{n}(u), z_{T(F)}\right)_{F}$, we infer that

$$
\mathcal{E}_{h}\left(\widehat{z}_{h}\right)=\mathcal{T}_{1}+\mathcal{T}_{2},
$$

where

$$
\begin{aligned}
\mathcal{T}_{1}:= & \sum_{T \in \mathcal{T}_{h}}\left(-\Delta u, z_{T}\right)_{T}+\sum_{F \in \mathcal{F}_{h}^{\mathrm{b}, \mathrm{D}} \cup \mathcal{F}_{h}^{\mathrm{b}, \mathrm{N}}}\left(\sigma_{n}(u), z_{F}\right)_{F}+\sum_{F \in \mathcal{F}_{h}^{\mathrm{b}, \mathrm{S}}}\left(\sigma_{n}(u), z_{T(F)}\right)_{F}-\sum_{T \in \mathcal{T}_{h}} \widehat{a}_{T}\left(I_{T}^{\backslash}(u), \widehat{z}_{T}\right), \\
\mathcal{T}_{2}:= & \sum_{F \in \mathcal{F}_{h}^{\mathrm{b}, \mathrm{S}}} \frac{1}{\gamma}\left(\sigma_{n}\left(E_{T(F)}^{\backslash}(u)\right), \sigma_{n}\left(R^{\backslash}\left(\widehat{z}_{T(F)}\right)\right)\right)_{F} \\
& -\sum_{F \in \mathcal{F}_{h}^{\mathrm{b}, \mathrm{S}}} \frac{1}{\gamma}\left(\left[\widehat{\phi}_{\gamma}\left(I_{T(F)}^{\backslash}(u)\right)\right]_{\mathbb{R}^{-}}, \widehat{\phi}_{\gamma}\left(\widehat{z}_{T(F)}\right)\right)_{F}-\sum_{F \in \mathcal{F}_{h}^{\mathrm{b}, \mathrm{S}}}\left(\sigma_{n}(u), z_{T(F)}\right)_{F},
\end{aligned}
$$

where we used in $\mathcal{T}_{1}$ that $z_{F}$ is zero for all $F \in \mathcal{F}_{h}^{\mathrm{b}, \mathrm{D}}$. We then observe that the term $\mathcal{T}_{1}$ can be rewritten by proceeding as in the proof of Lemma 5.4 (by letting $\mathcal{F}_{h}^{\mathrm{b}, \mathrm{S}}$ play the former role of $\mathcal{F}_{h}^{\mathrm{b}, \mathrm{D}}$ and $\mathcal{F}_{h}^{\mathrm{b}, \mathrm{D}} \cup \mathcal{F}_{h}^{\mathrm{b}, \mathrm{N}}$ 
play the former role of $\mathcal{F}_{h}^{\mathrm{b}, \mathrm{N}}$ ). We obtain

$$
\begin{aligned}
\mathcal{T}_{1}= & \sum_{T \in \mathcal{T}_{h}}\left(\nabla\left(u-E_{T}^{\backslash}(u)\right), \nabla z_{T}\right)_{T}-\sum_{T \in \mathcal{T}_{h}}\left(\sigma_{n}\left(u-E_{T}^{\backslash}(u)\right), z_{T}-z_{\partial T} \backslash\right)_{\partial T} \backslash \\
& -\sum_{T \in \mathcal{T}_{h}}\left(\eta_{\partial T} S^{\backslash}\left(I_{T}^{\searrow}(u)\right), S^{\backslash}\left(\widehat{z}_{T}\right)\right)_{\partial T} \backslash
\end{aligned}
$$

Hence, owing to the approximation results (5.7)-(5.8), we have

$$
\left|\mathcal{T}_{1}\right| \lesssim\left(\sum_{T \in \mathcal{T}_{h}}\left\|u-\pi_{T}^{k+1}(u)\right\|_{\sharp, T}^{2}\right)^{\frac{1}{2}}\left\|\widehat{z}_{h}\right\|_{\widehat{U}_{h, 0}^{k}} .
$$

Concerning $\mathcal{T}_{2}$, we use that $\sigma_{n}(u)=\left[\phi_{\gamma}(u)\right]_{\mathbb{R}^{-}}$, and re-arranging terms, we infer that $\mathcal{T}_{2}=-\mathcal{T}_{2,1}+\mathcal{T}_{2,2}$, where

$$
\begin{aligned}
& \mathcal{T}_{2,1}:=\sum_{F \in \mathcal{F}_{h}^{\mathrm{b}, \mathrm{S}}} \frac{1}{\gamma}\left(\sigma_{n}\left(u-E_{T(F)}^{\backslash}(u)\right), \sigma_{n}\left(R \backslash\left(\widehat{z}_{T(F)}\right)\right)\right)_{F}, \\
& \mathcal{T}_{2,2}:=\sum_{F \in \mathcal{F}_{h}^{\mathrm{b}, \mathrm{S}}} \frac{1}{\gamma}\left(\left[\phi_{\gamma}(u)\right]_{\mathbb{R}^{-}}-\left[\widehat{\phi}_{\gamma}\left(I_{T(F)}^{\backslash}(u)\right)\right]_{\mathbb{R}^{-}}, \widehat{\phi}_{\gamma}\left(\widehat{z}_{T(F)}\right)\right)_{F} .
\end{aligned}
$$

Recalling that $\gamma_{\mid F}=\gamma_{0} h_{F}^{-1}$, we can bound $\mathcal{T}_{2,1}$ using the approximation result (5.7) together with the discrete trace inequality from Lemma 3.1 as follows:

$$
\left|\mathcal{T}_{2,1}\right| \lesssim\left(\sum_{T \in \mathcal{T}_{h}}\left\|u-\pi_{T}^{k+1}(u)\right\|_{\sharp, T}^{2}\right)^{\frac{1}{2}}\left\|\widehat{z}_{h}\right\|_{\widehat{U}_{h, 0}^{k}} .
$$

Moreover, concerning $\mathcal{T}_{2,2}$, we have $\mathcal{T}_{2,2}=\mathcal{T}_{2,2,1}+\mathcal{T}_{2,2,2}+\mathcal{T}_{2,2,3}$, where

$$
\begin{aligned}
\mathcal{T}_{2,2,1} & :=\sum_{F \in \mathcal{F}_{h}^{\mathrm{b}, \mathrm{S}}} \frac{1}{\gamma}\left(\left[\widehat{\phi}_{\gamma}\left(\widehat{u}_{T(F)}\right)\right]_{\mathbb{R}^{-}}-\left[\widehat{\phi}_{\gamma}\left(I_{T(F)}^{\backslash}(u)\right)\right]_{\mathbb{R}^{-}}, \widehat{\phi}_{\gamma}\left(\widehat{z}_{T(F)}\right)\right)_{F}, \\
\mathcal{T}_{2,2,2} & :=\sum_{F \in \mathcal{F}_{h}^{\mathrm{b}, \mathrm{S}}} \frac{1}{\gamma}\left(\left[\phi_{\gamma}(u)\right]_{\mathbb{R}^{-}}-\left[\widehat{\phi}_{\gamma}\left(\widehat{u}_{T(F)}\right)\right]_{\mathbb{R}^{-}}, \widehat{\phi}_{\gamma}\left(\widehat{u}_{T(F)}\right)-\phi_{\gamma}(u)\right)_{F}, \\
\mathcal{T}_{2,2,3} & :=\sum_{F \in \mathcal{F}_{h}^{\mathrm{b}, \mathrm{S}}} \frac{1}{\gamma}\left(\left[\phi_{\gamma}(u)\right]_{\mathbb{R}^{-}}-\left[\widehat{\phi}_{\gamma}\left(\widehat{u}_{T(F)}\right)\right]_{\mathbb{R}^{-}}, \phi_{\gamma}(u)-\widehat{\phi}_{\gamma}\left(I_{T(F)}^{\backslash}(u)\right)\right)_{F} .
\end{aligned}
$$

We have $\mathcal{T}_{2,2,1}=\Delta \widehat{\phi}_{\gamma}\left(\widehat{u}_{h}, \widehat{I}_{h}(u)\right)$ and

$$
\mathcal{T}_{2,2,2} \leq-\sum_{F \in \mathcal{F}_{h}^{\mathrm{b}, \mathrm{S}}} \frac{1}{\gamma}\left\|\left[\phi_{\gamma}(u)\right]_{\mathbb{R}^{-}}-\left[\widehat{\phi}_{\gamma}\left(\widehat{u}_{T(F)}\right)\right]_{\mathbb{R}^{-}}\right\|_{F}^{2},
$$

where we used the identity (6.5). Moreover, using Young's inequality, we infer that

$$
\mathcal{T}_{2,2,3} \leq \sum_{F \in \mathcal{F}_{h}^{\mathrm{b}, \mathrm{S}}} \frac{1}{2 \gamma}\left\|\left[\phi_{\gamma}(u)\right]_{\mathbb{R}^{-}}-\left[\widehat{\phi}_{\gamma}\left(\widehat{u}_{T(F)}\right)\right]_{\mathbb{R}^{-}}\right\|_{F}^{2}+\sum_{F \in \mathcal{F}_{h}^{\mathrm{b}, \mathrm{S}}} \frac{2}{\gamma}\left\|\phi_{\gamma}(u)-\widehat{\phi}_{\gamma}\left(I_{T(F)}^{\backslash}(u)\right)\right\|_{F}^{2},
$$

and recalling the definitions of $\phi_{\gamma}, \widehat{\phi}_{\gamma}$, and $\gamma$, we have

$$
\begin{aligned}
\frac{2}{\gamma}\left\|\phi_{\gamma}(u)-\widehat{\phi}_{\gamma}\left(I_{T(F)}^{\backslash}(u)\right)\right\|_{F}^{2} & \lesssim h_{F}\left\|\boldsymbol{n}_{T(F)} \cdot \nabla\left(u-E_{T}^{\backslash}(u)\right)\right\|_{F}^{2}+h_{F}^{-1}\left\|u-\pi_{T(F)}^{k+1}(u)\right\|_{F}^{2} \\
& \lesssim\left\|u-\pi_{T(F)}^{k+1}(u)\right\|_{\sharp, T(F)}^{2},
\end{aligned}
$$


where we used the approximation result (5.7) to bound the first term on the right-hand side and the arguments in the proof of Lemma 5.1 to bound the second term (implying that $h_{F}^{-1}\left\|u-\pi_{T(F)}^{k+1}(u)\right\|_{F}^{2} \lesssim$ $\left.\left\|\nabla\left(u-\pi_{T(F)}^{k+1}(u)\right)\right\|_{T(F)}^{2} \lesssim\left\|u-\pi_{T(F)}^{k+1}(u)\right\|_{\sharp, T(F)}^{2}\right)$. Therefore, we obtain

$$
\mathcal{T}_{2,2,2}+\mathcal{T}_{2,2,3} \leq-\sum_{F \in \mathcal{F}_{h}^{\mathrm{b}, \mathrm{S}}} \frac{1}{2 \gamma}\left\|\left[\phi_{\gamma}(u)\right]_{\mathbb{R}^{-}}-\left[\widehat{\phi}_{\gamma}\left(\widehat{u}_{T(F)}\right)\right]_{\mathbb{R}^{-}}\right\|_{F}^{2}+C \sum_{T \in \mathcal{T}_{h}}\left\|u-\pi_{T}^{k+1}(u)\right\|_{\sharp, T}^{2} .
$$

Putting everything together, we obtain the expected estimate.

Theorem 6.4 ( $H^{1}$-error estimate). Assume that $u \in H^{1+s}(\Omega)$ with $s>\frac{1}{2}$. The following holds true:

$$
\sum_{T \in \mathcal{T}_{h}}\left\|\nabla\left(u-R^{\backslash}\left(\widehat{u}_{T}\right)\right)\right\|_{T}^{2}+\sum_{F \in \mathcal{F}_{h}^{\mathrm{b}, \mathrm{S}}} h_{T(F)}\left\|\left[\phi_{\gamma}(u)\right]_{\mathbb{R}^{-}}-\left[\widehat{\phi}_{\gamma}\left(\widehat{u}_{T(F)}\right)\right]_{\mathbb{R}^{-}}\right\|_{F}^{2} \lesssim \sum_{T \in \mathcal{T}_{h}}\left\|u-\pi_{T}^{k+1}(u)\right\|_{\sharp, T}^{2} .
$$

Consequently, letting $t:=\min (k+1, s)$, we have

$$
\sum_{T \in \mathcal{T}_{h}}\left\|\nabla\left(u-R \backslash\left(\widehat{u}_{T}\right)\right)\right\|_{T}^{2}+\sum_{F \in \mathcal{F}_{h}^{\mathrm{b}, \mathrm{S}}} h_{T(F)}\left\|\left[\phi_{\gamma}(u)\right]_{\mathbb{R}^{-}}-\left[\widehat{\phi}_{\gamma}\left(\widehat{u}_{T(F)}\right)\right]_{\mathbb{R}^{-}}\right\|_{F}^{2} \lesssim \sum_{T \in \mathcal{T}_{h}} h_{T}^{2 t}|u|_{H^{2+}(T)}^{2} .
$$

Proof. Recall that $\widehat{z}_{h}=\widehat{u}_{h}-\widehat{I}_{h}(u)$. Owing to the monotonicity property (6.6), the definition of the consistency error, and the bound from Lemma 6.3, we infer that

$$
\begin{aligned}
& \alpha\left\|\widehat{z}_{h}\right\|_{\widehat{U}_{h, 0}^{k}}^{2}+\Delta \widehat{\phi}_{\gamma}\left(\widehat{u}_{h}, \widehat{I}_{h}(u)\right)+\sum_{F \in \mathcal{F}_{h}^{\mathrm{b}, \mathrm{S}}} \frac{1}{2 \gamma}\left\|\left[\phi_{\gamma}(u)\right]_{\mathbb{R}^{-}}-\left[\widehat{\phi}_{\gamma}\left(\widehat{u}_{T(F)}\right)\right]_{\mathbb{R}^{-}}\right\|_{F}^{2} \\
& \leq \mathcal{E}_{h}\left(\widehat{z}_{h}\right)+\sum_{F \in \mathcal{F}_{h}^{\mathrm{b}, \mathrm{S}}} \frac{1}{2 \gamma}\left\|\left[\phi_{\gamma}(u)\right]_{\mathbb{R}^{-}}-\left[\widehat{\phi}_{\gamma}\left(\widehat{u}_{T(F)}\right)\right]_{\mathbb{R}^{-}}\right\|_{F}^{2} \\
& \lesssim\left(\sum_{T \in \mathcal{T}_{h}}\left\|u-\pi_{T}^{k+1}(u)\right\|_{\sharp, T}^{2}\right)^{\frac{1}{2}}\left\|\widehat{z}_{h}\right\|_{\widehat{U}_{h, 0}^{k}}+\Delta \widehat{\phi}_{\gamma}\left(\widehat{u}_{h}, \widehat{I}_{h}(u)\right)+C \sum_{T \in \mathcal{T}_{h}}\left\|u-\pi_{T}^{k+1}(u)\right\|_{\sharp, T}^{2} .
\end{aligned}
$$

Hence, clearing the term $\Delta \widehat{\phi}_{\gamma}\left(\widehat{u}_{h}, \widehat{I}_{h}(u)\right)$ and invoking Young's inequality leads to the bound (6.11). Finally, (6.12) follows from (6.11) and Lemma 3.4.

Remark 6.5 (Face version). The main bottleneck when considering the face version of the Nitsche-HHO method is to bound the term $h_{F}\left\|\phi_{\gamma}(u)-\widehat{\phi}_{\gamma}\left(I_{T(F)}^{\backslash}(u)\right)\right\|_{F}^{2}$ when estimating $\mathcal{T}_{2,2,3}$. Indeed, in this case, we end up with a term of the form $h_{F}^{-1}\left\|u-\pi_{F}^{k}(u)\right\|_{F}^{2}$, which is of order $\mathcal{O}\left(h^{2 k}\right)$, instead of $h_{F}^{-1}\left\|u-\pi_{T(F)}^{k+1}(u)\right\|_{F}^{2}$, which is of order $\mathcal{O}\left(h^{2(k+1)}\right)$. Thus, the above analysis for the face version leads to a suboptimal $H^{1}$-error estimate of order $\mathcal{O}\left(h^{k}\right)$. Our numerical experiments, yet, indicate that one can still hope for the optimal rate $\mathcal{O}\left(h^{k+1}\right)$.

\section{Numerical results}

All the numerical computations are performed using the Open-Source diskpp library ${ }^{1}$ [23]. We consider uniformly refined sequences of triangular meshes and of hexagonal meshes to illustrate the polyhedral capabilities of Nitsche-HHO.

\subsection{Dirichlet conditions}

We consider the Poisson model problem (2.1). The domain $\Omega$ is the unit square. The numerical results are compared to the closed-form solution $u(x, y)=\cos (\pi x) \cos (\pi y)$ corresponding to the right-hand side $f(x, y)=2 \pi^{2} \cos (\pi x) \cos (\pi y)$ and satisfying a homogeneous Dirichlet condition over the whole boundary, i.e. we set $\Gamma_{D}=\partial \Omega$. The errors in the $H^{1}$-norm and the convergence rates are reported in Table 1 using 
Table 1: $H^{1}$-error and convergence rates for Dirichlet conditions. Face version with $\theta=1, \gamma_{0}=5$ and $k \in\{0,1,2,3\}$.

\begin{tabular}{|c|c|c|c|c|c|c|c|c|}
\hline \multicolumn{9}{|c|}{ Triangles } \\
\hline \multirow[b]{2}{*}{$h$} & \multicolumn{2}{|c|}{$k=0$} & \multicolumn{2}{|c|}{$k=1$} & \multicolumn{2}{|c|}{$k=2$} & \multicolumn{2}{|c|}{$k=3$} \\
\hline & error & rate & error & rate & error & rate & error & rate \\
\hline 0.230 & 7.183e-01 & & $9.291 \mathrm{e}-02$ & & $9.617 \mathrm{e}-03$ & & $5.278 \mathrm{e}-04$ & \\
\hline 0.115 & $3.418 \mathrm{e}-01$ & 1.071 & $2.399 \mathrm{e}-02$ & 1.953 & $1.241 \mathrm{e}-03$ & 2.955 & $3.457 \mathrm{e}-05$ & 3.932 \\
\hline 0.057 & $1.665 \mathrm{e}-01$ & 1.037 & $6.081 \mathrm{e}-03$ & 1.980 & $1.569 \mathrm{e}-04$ & 2.983 & $2.205 \mathrm{e}-06$ & 3.971 \\
\hline 0.029 & $8.217 \mathrm{e}-02$ & 1.019 & $1.530 \mathrm{e}-03$ & 1.991 & $1.971 \mathrm{e}-05$ & 2.993 & $1.391 \mathrm{e}-07$ & 3.986 \\
\hline \multicolumn{9}{|c|}{ Hexagons } \\
\hline & \multicolumn{2}{|c|}{$k=0$} & \multicolumn{2}{|c|}{$k=1$} & \multicolumn{2}{|c|}{$k=2$} & \multicolumn{2}{|c|}{$k=3$} \\
\hline$h$ & error & rate & error & rate & error & rate & error & rate \\
\hline 0.176 & $5.385 \mathrm{e}-01$ & & $5.005 \mathrm{e}-02$ & & $3.244 \mathrm{e}-03$ & & $1.438 \mathrm{e}-04$ & \\
\hline 0.091 & $2.633 \mathrm{e}-01$ & 1.078 & $1.277 \mathrm{e}-02$ & 2.059 & $4.183 \mathrm{e}-04$ & 3.088 & $9.295 \mathrm{e}-06$ & 4.129 \\
\hline 0.046 & $1.299 \mathrm{e}-01$ & 1.044 & $3.216 \mathrm{e}-03$ & 2.038 & $5.303 \mathrm{e}-05$ & 3.052 & $5.898 \mathrm{e}-07$ & 4.075 \\
\hline 0.023 & $6.446 \mathrm{e}-02$ & 1.023 & $8.065 \mathrm{e}-04$ & 2.021 & $6.674 \mathrm{e}-06$ & 3.028 & $3.713 \mathrm{e}-08$ & 4.040 \\
\hline
\end{tabular}

the face version of Nitsche-HHO. We observe convergence rates that match those predicted by the theory (Theorem 4.7).

We also study the symmetric and nonsymmetric variants of the cell version of Nitsche-HHO. The errors in the $H^{1}$-norm and the convergence rates for the symmetric variant $\theta=1$, with $\gamma_{0}=5$, are reported in Table 2. These results confirm the predictions from Theorem 5.5. The numerical results for the nonsymmetric variants are reported in Table $3\left(\theta=0\right.$ and $\left.\gamma_{0}=1\right)$ and Table $4\left(\theta=-1\right.$ and $\left.\gamma_{0}=0\right)$. Notice that for the skew-symmetric method $(\theta=-1)$, the penalty-free variant $\left(\gamma_{0}=0\right)$ is chosen and optimal rates are still observed. Although the analysis presented in this paper does not cover this case, our results are in agreement with the analysis presented in [12] for Lagrange and Crouzeix-Raviart finite elements. Moreover, we performed convergence tests for the equal-order case $(l=k)$, and suboptimal convergence rates were observed (as expected). We do not present these results for the sake of brevity.

Table 2: $H^{1}$-error and convergence rates for Dirichlet conditions. Cell version with $\theta=1, \gamma_{0}=5$ and $k \in\{0,1,2,3\}$.

\begin{tabular}{|c|c|c|c|c|c|c|c|c|}
\hline \multicolumn{9}{|c|}{ Triangles } \\
\hline \multirow[b]{2}{*}{$h$} & \multicolumn{2}{|c|}{$k=0$} & \multicolumn{2}{|c|}{$k=1$} & \multicolumn{2}{|c|}{$k=2$} & \multicolumn{2}{|c|}{$k=3$} \\
\hline & error & rate & error & rate & error & rate & error & rate \\
\hline 0.230 & $6.179 \mathrm{e}-01$ & & $9.071 \mathrm{e}-02$ & & $9.739 \mathrm{e}-03$ & & $5.222 \mathrm{e}-04$ & \\
\hline 0.115 & $3.188 \mathrm{e}-01$ & 0.954 & $2.442 \mathrm{e}-02$ & 1.893 & $1.322 \mathrm{e}-03$ & 2.882 & $3.570 \mathrm{e}-05$ & 3.87166 \\
\hline 0.057 & $1.615 \mathrm{e}-01$ & 0.982 & $6.323 \mathrm{e}-03$ & 1.950 & $1.708 \mathrm{e}-04$ & 2.952 & $2.330 \mathrm{e}-06$ & 3.93836 \\
\hline 0.029 & $8.118 \mathrm{e}-02$ & 0.992 & $1.608 \mathrm{e}-03$ & 1.976 & $2.167 \mathrm{e}-05$ & 2.978 & $1.487 \mathrm{e}-07$ & 3.97069 \\
\hline \multicolumn{9}{|c|}{ Hexagons } \\
\hline & \multicolumn{2}{|c|}{$k=0$} & \multicolumn{2}{|c|}{$k=1$} & \multicolumn{2}{|c|}{$k=2$} & \multicolumn{2}{|c|}{$k=3$} \\
\hline$h$ & error & rate & error & rate & error & rate & error & rate \\
\hline 0.176 & $4.895 \mathrm{e}-01$ & & $4.723 \mathrm{e}-02$ & & $3.150 \mathrm{e}-03$ & & $1.402 \mathrm{e}-04$ & \\
\hline 0.091 & $2.514 \mathrm{e}-01$ & 1.004 & $1.239 \mathrm{e}-02$ & 2.017 & $4.129 \mathrm{e}-04$ & 3.063 & $9.184 \mathrm{e}-06$ & 4.108 \\
\hline 0.046 & $1.270 \mathrm{e}-01$ & 1.010 & $3.168 \mathrm{e}-03$ & 2.015 & $5.271 \mathrm{e}-05$ & 3.042 & $5.864 \mathrm{e}-07$ & 4.066 \\
\hline 0.023 & $6.375 \mathrm{e}-02$ & 1.007 & $8.005 \mathrm{e}-04$ & 2.010 & $6.654 \mathrm{e}-06$ & 3.024 & $3.702 \mathrm{e}-08$ & 4.036 \\
\hline
\end{tabular}

\footnotetext{
${ }^{1}$ https://github.com/wareHHOuse/diskpp
} 
Table 3: $H^{1}$-error and convergence rates for Dirichlet conditions. Cell version with $\theta=0, \gamma_{0}=1$ and $k \in\{0,1,2,3\}$.

\begin{tabular}{|c|c|c|c|c|c|c|c|c|}
\hline \multicolumn{9}{|c|}{ Triangles } \\
\hline \multirow[b]{2}{*}{$h$} & \multicolumn{2}{|c|}{$k=0$} & \multicolumn{2}{|c|}{$k=1$} & \multicolumn{2}{|c|}{$k=2$} & \multicolumn{2}{|c|}{$k=3$} \\
\hline & error & rate & error & rate & error & rate & error & rate \\
\hline 0.230 & $7.312 \mathrm{e}-01$ & & $9.482 \mathrm{e}-02$ & & $1.128 \mathrm{e}-02$ & & $6.219 \mathrm{e}-04$ & \\
\hline 0.115 & $3.453 \mathrm{e}-01$ & 1.083 & $2.486 \mathrm{e}-02$ & 1.931 & $1.409 \mathrm{e}-03$ & 3.001 & $3.942 \mathrm{e}-05$ & 3.979 \\
\hline 0.057 & $1.678 \mathrm{e}-01$ & 1.041 & $6.375 \mathrm{e}-03$ & 1.963 & $1.760 \mathrm{e}-04$ & 3.001 & $2.456 \mathrm{e}-06$ & 4.004 \\
\hline 0.029 & $8.271 \mathrm{e}-02$ & 1.020 & $1.614 \mathrm{e}-03$ & 1.982 & $2.199 \mathrm{e}-05$ & 3.001 & $1.528 \mathrm{e}-07$ & 4.007 \\
\hline \multicolumn{9}{|c|}{ Hexagons } \\
\hline & \multicolumn{2}{|c|}{$k=0$} & \multicolumn{2}{|c|}{$k=1$} & \multicolumn{2}{|c|}{$k=2$} & \multicolumn{2}{|c|}{$k=3$} \\
\hline$h$ & error & rate & error & rate & error & rate & error & rate \\
\hline 0.176 & $5.184 \mathrm{e}-01$ & & $5.140 \mathrm{e}-02$ & & $3.269 \mathrm{e}-03$ & & $1.448 \mathrm{e}-04$ & \\
\hline 0.091 & $2.582 \mathrm{e}-01$ & 1.050 & $1.291 \mathrm{e}-02$ & 2.083 & $4.196 \mathrm{e}-04$ & 3.094 & $9.321 \mathrm{e}-06$ & 4.134 \\
\hline 0.046 & $1.286 \mathrm{e}-01$ & 1.030 & $3.232 \mathrm{e}-03$ & 2.047 & $5.311 \mathrm{e}-05$ & 3.055 & $5.906 \mathrm{e}-07$ & 4.077 \\
\hline 0.023 & $6.414 \mathrm{e}-02$ & 1.016 & $8.086 \mathrm{e}-04$ & 2.025 & $6.678 \mathrm{e}-06$ & 3.029 & $3.715 \mathrm{e}-08$ & 4.041 \\
\hline
\end{tabular}

Table 4: $H^{1}$-error and convergence rates for Dirichlet conditions. Cell version with $\theta=-1, \gamma_{0}=0$ and $k \in\{0,1,2,3\}$.

\begin{tabular}{|c|c|c|c|c|c|c|c|c|}
\hline \multicolumn{9}{|c|}{ Triangles } \\
\hline \multirow[b]{2}{*}{$h$} & \multicolumn{2}{|c|}{$k=0$} & \multicolumn{2}{|c|}{$k=1$} & \multicolumn{2}{|c|}{$k=2$} & \multicolumn{2}{|c|}{$k=3$} \\
\hline & error & rate & error & rate & error & rate & error & rate \\
\hline 0.230 & $6.767 \mathrm{e}-01$ & & $9.044 \mathrm{e}-02$ & & $1.019 \mathrm{e}-02$ & & $5.403 \mathrm{e}-04$ & \\
\hline 0.115 & $3.322 \mathrm{e}-01$ & 1.026 & $2.426 \mathrm{e}-02$ & 1.898 & $1.346 \mathrm{e}-03$ & 2.920 & $3.635 \mathrm{e}-05$ & 3.893 \\
\hline 0.057 & $1.646 \mathrm{e}-01$ & 1.013 & $6.298 \mathrm{e}-03$ & 1.946 & $1.722 \mathrm{e}-04$ & 2.967 & $2.352 \mathrm{e}-06$ & 3.950 \\
\hline 0.029 & $8.193 \mathrm{e}-02$ & 1.006 & $1.604 \mathrm{e}-03$ & 1.973 & $2.176 \mathrm{e}-05$ & 2.985 & $1.494 \mathrm{e}-07$ & 3.977 \\
\hline \multicolumn{9}{|c|}{ Hexagons } \\
\hline & \multicolumn{2}{|c|}{$k=0$} & \multicolumn{2}{|c|}{$k=1$} & \multicolumn{2}{|c|}{$k=2$} & \multicolumn{2}{|c|}{$k=3$} \\
\hline$h$ & error & rate & error & rate & error & rate & error & rate \\
\hline 0.176 & $5.069 \mathrm{e}-01$ & & $5.029 \mathrm{e}-02$ & & $3.213 \mathrm{e}-03$ & & $1.416 \mathrm{e}-04$ & \\
\hline 0.091 & $2.553 \mathrm{e}-01$ & 1.034 & $1.278 \mathrm{e}-02$ & 2.065 & $4.165 \mathrm{e}-04$ & 3.079 & $9.227 \mathrm{e}-06$ & 4.117 \\
\hline 0.046 & $1.279 \mathrm{e}-01$ & 1.022 & $3.216 \mathrm{e}-03$ & 2.039 & $5.293 \mathrm{e}-05$ & 3.049 & $5.877 \mathrm{e}-07$ & 4.069 \\
\hline 0.023 & $6.397 \mathrm{e}-02$ & 1.012 & $8.065 \mathrm{e}-04$ & 2.021 & $6.668 \mathrm{e}-06$ & 3.027 & $3.706 \mathrm{e}-08$ & 4.038 \\
\hline
\end{tabular}




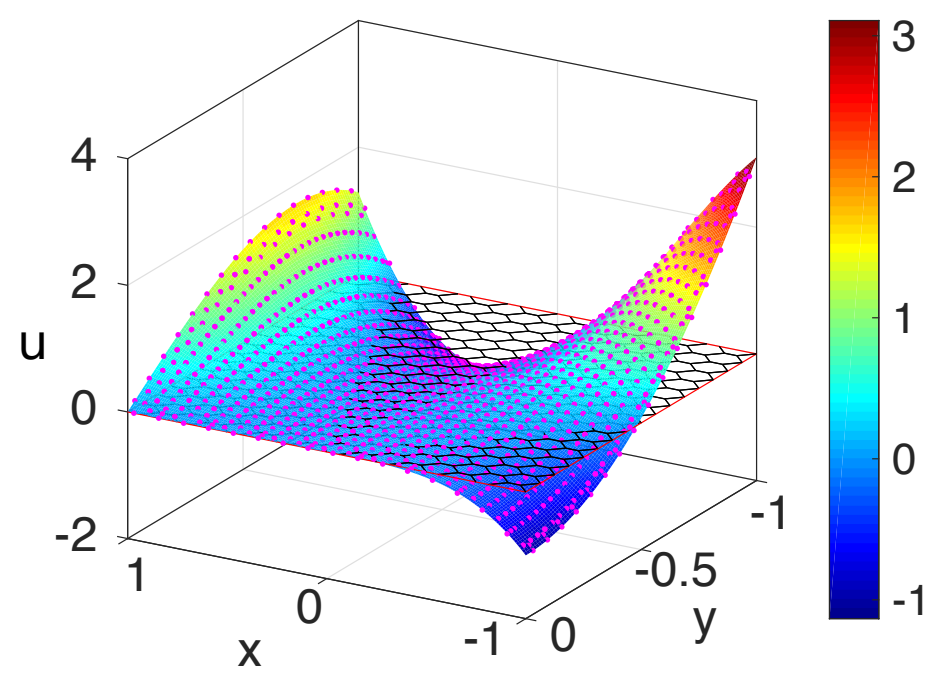

Figure 1: Exact solution for Signorini conditions and test case 1. The magenta dots represent the values of the numerical solution at the faces and cells barycenters of an hexagonal mesh (depicted in black). The contact boundary corresponds to the side $\{y=0\}$.

\subsection{Signorini conditions}

We present here two test cases for Signorini conditions. We consider the model problem (2.4)-(2.5) in two dimensions. To deal with the nonlinearity, we use a semi-smooth Newton solver [3, 49, 56].

\subsubsection{Test case 1: manufactured solution}

We build an exact solution using polar coordinates for the model problem $(2.4)-(2.5)$, defined in $\Omega=$ $[-1,1] \times[-1,0]$. The closed form solution $u: \Omega \rightarrow \mathbb{R}^{2}$ in polar coordinates is

$$
u(r, \theta)=-r^{\frac{11}{2}} \sin \left(\frac{11}{2} \theta\right)
$$

with a source term $f=0$. The Signorini boundary is located at the top of the domain $\Gamma_{S}=[-1,1] \times\{0\}$, and the transition between biding and nonbiding happens at $(0,0)$. Appropriate Dirichlet conditions are applied on the remaining boundaries. The exact solution is depicted in Figure 1 along with the numerical solution at each barycenter of faces and cells of an hexagonal mesh.

We present in Table 5 and Table 6 the errors in the $H^{1}$-norm and convergence rates using the symmetric variant of Nitsche-HHO $(\theta=1)$ and polynomial degrees up to 3 , for the face and the cell versions, respectively. Notice that the analytical solution (7.1) enjoys the needed regularity to expect optimal convergence rates for these polynomial orders, even if the transition point $(0,0)$ is not a node of the mesh. We report the numerical results for the nonsymmetric variants of the cell version in Table $7(\theta=0)$ and Table 8 $(\theta=-1)$. In order to be consistent with the theoretical lower bound on the penalty parameter, we use $\gamma_{0}=(k+1)(k+2)$ for $\theta=1$ and $\gamma_{0}=\frac{1}{4}(k+1)(k+2)$ for $\theta=0$ (these values correspond to the estimation of the constant $C_{\mathrm{dt}}$ in Lemma 3.1, see [64, Theorem 3] for triangles). In all cases, the numerical results are in good agreement with the expected asymptotic convergence rates, even for the face version.

We display in Figure 2 and Figure 3 the number of iterations as a function of the penalty parameter $\gamma_{0}$ with $k \in\{0,1,2,3\}$ and $\theta=-1$ (left panel) or $\theta=1$ (right panel). We set a residual convergence threshold of $10^{-9}$ for the semi-smooth Newton solver. We run our test over a coarse uniform triangulation with meshsize $h=0.168$ and a coarse hexagonal mesh with meshsize $h=0.622$. For the value $\theta=1$ on hexagonal meshes, we observed a severe degradation on the semi-smooth Newton convergence for the lowest values of $\gamma_{0}$, and therefore the corresponding results are not reported. This emphasizes the role of the shape of the cells on the constant $C_{d t}$ involved in Lemma 6.1. A bit surprisingly, these difficulties were 
Table 5: Test case 1 for Signorini conditions. $H^{1}$-error and convergence rates for the face version with $\theta=1$, $\gamma_{0}=(k+1)(k+2)$ and $k \in\{0,1,2,3\}$.

\begin{tabular}{|c|c|c|c|c|c|c|c|c|}
\hline \multicolumn{9}{|c|}{ Triangles } \\
\hline \multirow[b]{2}{*}{$h$} & \multicolumn{2}{|c|}{$k=0$} & \multicolumn{2}{|c|}{$k=1$} & \multicolumn{2}{|c|}{$k=2$} & \multicolumn{2}{|c|}{$k=3$} \\
\hline & error & rate & error & rate & error & rate & error & rate \\
\hline 0.168 & $1.311 \mathrm{e}+00$ & & $1.727 \mathrm{e}-01$ & & $1.68 \mathrm{e}-02$ & & $2.606 \mathrm{e}-04$ & \\
\hline 0.084 & $7.144 \mathrm{e}-01$ & 0.876 & $4.588 \mathrm{e}-02$ & 1.912 & $1.403 \mathrm{e}-03$ & 3.058 & $1.667 \mathrm{e}-05$ & 3.966 \\
\hline 0.042 & $3.741 \mathrm{e}-01$ & 0.933 & $1.186 \mathrm{e}-02$ & 1.951 & $1.654 \mathrm{e}-04$ & 3.084 & $1.054 \mathrm{e}-06$ & 3.984 \\
\hline \multicolumn{9}{|c|}{ Hexagons } \\
\hline & \multicolumn{2}{|c|}{$k=0$} & \multicolumn{2}{|c|}{$k=1$} & \multicolumn{2}{|c|}{$k=2$} & \multicolumn{2}{|c|}{$k=3$} \\
\hline$h$ & error & rate & error & rate & error & rate & error & rate \\
\hline 0.622 & $3.621 \mathrm{e}+00$ & & $1.576 \mathrm{e}+00$ & & $2.262 \mathrm{e}-01$ & & $2.517 \mathrm{e}-02$ & \\
\hline 0.338 & $2.564 \mathrm{e}+00$ & 0.566 & $4.494 \mathrm{e}-01$ & 2.058 & $3.973 \mathrm{e}-02$ & 3.327 & $1.809 \mathrm{e}-03$ & 4.342 \\
\hline 0.177 & $1.508 \mathrm{e}+00$ & 0.824 & $1.190 \mathrm{e}-01$ & 2.063 & $3.814 \mathrm{e}-03$ & 3.188 & $1.214 \mathrm{e}-04$ & 4.203 \\
\hline 0.091 & $8.179 \mathrm{e}-01$ & 0.918 & $3.057 \mathrm{e}-02$ & 2.039 & $4.835 \mathrm{e}-04$ & 3.100 & $7.856 \mathrm{e}-06$ & 4.112 \\
\hline
\end{tabular}

Table 6: Test case 1 for Signorini conditions. $H^{1}$-error and convergence rates for the cell version with $\theta=1$, $\gamma_{0}=(k+1)(k+2)$ and $k \in\{0,1,2,3\}$.

\begin{tabular}{|c|c|c|c|c|c|c|c|c|}
\hline \multicolumn{9}{|c|}{ Triangles } \\
\hline \multirow[b]{2}{*}{$h$} & \multicolumn{2}{|c|}{$k=0$} & \multicolumn{2}{|c|}{$k=1$} & \multicolumn{2}{|c|}{$k=2$} & \multicolumn{2}{|c|}{$k=3$} \\
\hline & error & rate & error & rate & error & rate & error & rate \\
\hline 0.168 & $1.338 \mathrm{e}+00$ & & $1.948 \mathrm{e}-01$ & & $1.377 \mathrm{e}-02$ & & $2.937 \mathrm{e}-04$ & \\
\hline 0.084 & $7.268 \mathrm{e}-01$ & 0.880 & $5.210 \mathrm{e}-02$ & 1.903 & $1.789 \mathrm{e}-03$ & 2.944 & $1.917 \mathrm{e}-05$ & 3.938 \\
\hline 0.042 & $3.802 \mathrm{e}-01$ & 0.935 & $1.356 \mathrm{e}-02$ & 1.942 & $2.220 \mathrm{e}-04$ & 3.010 & $1.220 \mathrm{e}-06$ & 3.973 \\
\hline \multicolumn{9}{|c|}{ Hexagons } \\
\hline & \multicolumn{2}{|c|}{$k=0$} & \multicolumn{2}{|c|}{$k=1$} & \multicolumn{2}{|c|}{$k=2$} & \multicolumn{2}{|c|}{$k=3$} \\
\hline$h$ & error & rate & error & rate & error & rate & error & rate \\
\hline 0.622 & $3.708 \mathrm{e}+00$ & & $1.719 \mathrm{e}+00$ & & $2.2488 \mathrm{e}-01$ & & $2.582 \mathrm{e}-02$ & \\
\hline 0.338 & $2.613 \mathrm{e}+00$ & 0.574 & $4.775 \mathrm{e}-01$ & 2.100 & $3.142 \mathrm{e}-02$ & 3.393 & $1.828 \mathrm{e}-03$ & 4.342 \\
\hline 0.177 & $1.525 \mathrm{e}+00$ & 0.837 & $1.233 \mathrm{e}-01$ & 2.101 & $3.927 \mathrm{e}-03$ & 3.229 & $1.219 \mathrm{e}-04$ & 4.203 \\
\hline 0.091 & $8.226 \mathrm{e}-01$ & 0.926 & $3.117 \mathrm{e}-02$ & 2.064 & $4.907 \mathrm{e}-04$ & 3.121 & $7.872 \mathrm{e}-06$ & 4.112 \\
\hline
\end{tabular}

Table 7: Test case 1 for Signorini conditions. $H^{1}$-error and convergence rates for the cell version with $\theta=0$, $\gamma_{0}=\frac{1}{4}(k+1)(k+2)$ and $k \in\{0,1,2,3\}$.

\begin{tabular}{|c|c|c|c|c|c|c|c|c|}
\hline \multicolumn{9}{|c|}{ Triangles } \\
\hline \multirow[b]{2}{*}{$h$} & \multicolumn{2}{|l|}{$k=0$} & \multicolumn{2}{|c|}{$k=1$} & \multicolumn{2}{|c|}{$k=2$} & \multicolumn{2}{|c|}{$k=3$} \\
\hline & error & rate & error & rate & error & rate & error & rate \\
\hline 0.168 & $1.338 \mathrm{e}+00$ & & $1.944 \mathrm{e}-01$ & & $9.741 \mathrm{e}-03$ & & $2.880 \mathrm{e}-04$ & \\
\hline 0.084 & $7.271 \mathrm{e}-01$ & 0.880 & $5.206 \mathrm{e}-02$ & 1.901 & $1.265 \mathrm{e}-03$ & 2.945 & $1.896 \mathrm{e}-05$ & 3.925 \\
\hline 0.042 & $3.803 \mathrm{e}-01$ & 0.935 & $1.355 \mathrm{e}-02$ & 1.942 & $1.609 \mathrm{e}-04$ & 2.975 & $1.214 \mathrm{e}-06$ & 3.964 \\
\hline \multicolumn{9}{|c|}{ Hexagons } \\
\hline & \multicolumn{2}{|l|}{$k=0$} & \multicolumn{2}{|c|}{$k=1$} & \multicolumn{2}{|c|}{$k=2$} & \multicolumn{2}{|c|}{$k=3$} \\
\hline$h$ & error & rate & error & rate & error & rate & error & rate \\
\hline 0.622 & $3.663 \mathrm{e}+00$ & & $1.719 \mathrm{e}+00$ & & $2.478 \mathrm{e}-01$ & & $2.526 \mathrm{e}-02$ & \\
\hline 0.338 & $2.604 \mathrm{e}+00$ & 0.560 & $4.773 \mathrm{e}-01$ & 2.101 & $3.129 \mathrm{e}-02$ & 3.393 & $1.815 \mathrm{e}-03$ & 4.317 \\
\hline 0.177 & $1.522 \mathrm{e}+00$ & 0.834 & $1.233 \mathrm{e}-01$ & 2.101 & $3.918 \mathrm{e}-03$ & 3.226 & $1.216 \mathrm{e}-04$ & 4.197 \\
\hline 0.091 & $8.218 \mathrm{e}-01$ & 0.924 & $3.117 \mathrm{e}-02$ & 2.064 & $4.901 \mathrm{e}-04$ & 3.119 & $7.864 \mathrm{e}-06$ & 4.109 \\
\hline
\end{tabular}


Table 8: Test case 1 for Signorini conditions. $H^{1}$-error and convergence rates for the cell version with

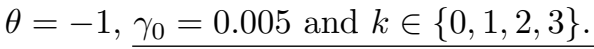

\begin{tabular}{|c|c|c|c|c|c|c|c|c|}
\hline \multicolumn{9}{|c|}{ Triangles } \\
\hline \multirow[b]{2}{*}{$h$} & \multicolumn{2}{|c|}{$k=0$} & \multicolumn{2}{|c|}{$k=1$} & \multicolumn{2}{|c|}{$k=2$} & \multicolumn{2}{|c|}{$k=3$} \\
\hline & error & rate & error & rate & error & rate & error & rate \\
\hline 0.168 & $1.311 \mathrm{e}+00$ & & $1.733 \mathrm{e}-01$ & & $8.645 \mathrm{e}-03$ & & $1.801 \mathrm{e}-04$ & \\
\hline 0.084 & $7.144 \mathrm{e}-01$ & 0.876 & $4.601 \mathrm{e}-02$ & 1.931 & $1.105 \mathrm{e}-03$ & 2.968 & $1.149 \mathrm{e}-05$ & 3.970 \\
\hline 0.042 & $3.741 \mathrm{e}-01$ & 0.933 & $1.189 \mathrm{e}-02$ & 1.953 & $1.391 \mathrm{e}-04$ & 2.989 & $7.231 \mathrm{e}-07$ & 3.990 \\
\hline \multicolumn{9}{|c|}{ Hexagons } \\
\hline & \multicolumn{2}{|c|}{$k=0$} & \multicolumn{2}{|c|}{$k=1$} & \multicolumn{2}{|c|}{$k=2$} & \multicolumn{2}{|c|}{$k=3$} \\
\hline$h$ & error & rate & error & rate & error & rate & error & rate \\
\hline 0.622 & $3.640 \mathrm{e}+00$ & & $1.721 \mathrm{e}+00$ & & $2.487 \mathrm{e}-01$ & & $2.538 \mathrm{e}-02$ & \\
\hline 0.338 & $2.599 \mathrm{e}+00$ & 0.552 & $4.780 \mathrm{e}-01$ & 2.100 & $3.151 \mathrm{e}-02$ & 3.387 & $1.824 \mathrm{e}-03$ & 4.317 \\
\hline 0.177 & $1.522 \mathrm{e}+00$ & 0.831 & $1.235 \mathrm{e}-01$ & 2.101 & $3.943 \mathrm{e}-03$ & 3.227 & $1.219 \mathrm{e}-04$ & 4.200 \\
\hline 0.091 & $8.221 \mathrm{e}-01$ & 0.924 & $3.121 \mathrm{e}-02$ & 2.065 & $4.920 \mathrm{e}-04$ & 3.123 & $7.875 \mathrm{e}-06$ & 4.111 \\
\hline
\end{tabular}

not encountered on triangular meshes. Also, whenever convergence is achieved, the number of semi-smooth Newton iterations is almost independent of $\theta$, and, as expected, depends mostly on the polynomial order $k$ and on the value of $\gamma_{0}$, whereby larger values of $k$ and of $\gamma_{0}$ lead to an increase of the number of iterations. In all cases, though, the number of iterations remains within reasonable values. Concerning the relative $H^{1}$-errors, we compute them using the term $\left(\sum_{T \in \mathcal{T}_{h}}\left\|\nabla\left(R \backslash \circ I_{T}^{\backslash}(u)\right)\right\|_{T}^{2}\right)^{\frac{1}{2}}$ for normalization. The relative $H^{1}$-errors turn out to be fairly independent of the value of $\gamma_{0}$ (whenever convergence is achieved). The values of the $H^{1}$-errors are $5.285 \cdot 10^{-1}, 2.271 \cdot 10^{-1}, 3.255 \cdot 10^{-2}, 3.771 \cdot 10^{-5}$, for $k \in\{0,1,2,3\}$ and $\theta=-1$, and $1.773 \cdot 10^{-1}, 2.580 \cdot 10^{-2}, 1.062 \cdot 10^{-1}, 3.308 \cdot 10^{-3}$, for $k \in\{0,1,2,3\}$ and $\theta=1$.
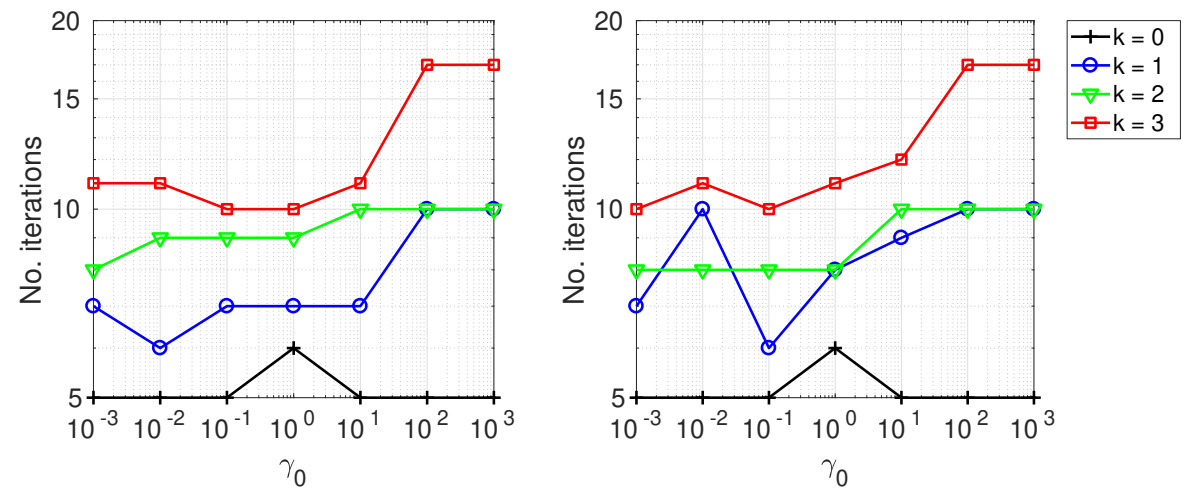

Figure 2: Influence of the penalty parameter $\gamma_{0}$ on the number of semi-smooth Newton iterations. Triangular mesh with size $h=0.168, \theta=-1$ (left) and $\theta=1$ (right), $k \in\{0,1,2,3\}$.

\subsubsection{Test case 2}

We now consider a test case described in [5] (see also [15]) on the unit square $\Omega=(0,1)^{2}$. The contact boundary is located at the bottom of the domain $\Gamma_{S}=[0,1] \times\{0\}$, whereas an homogeneous Dirichlet condition is applied at the top boundary $\Gamma_{D}=[0,1] \times\{1\}$. On the remaining parts of the boundary, homogeneous Neumann boundary conditions are applied, i.e. $\Gamma_{N}=(\{0\} \times[0,1]) \cup(\{1\} \times[0,1])$. The expression for the source term is $f=2 \pi \sin (2 \pi x)$.

There is no closed-form solution to this problem up to our knowledge, so that the reference solution is computed using a very fine triangulation with mesh size $h=0.005$ and with quadratic and cubic polynomials on the faces and in the cells, respectively. A transition between biding and nonbiding on $\Gamma_{S}$ has been reported numerically in $[5,15]$, as well as optimal convergence rates in $L^{2}$ - and $H^{1}$-norms for piecewise linear finite 

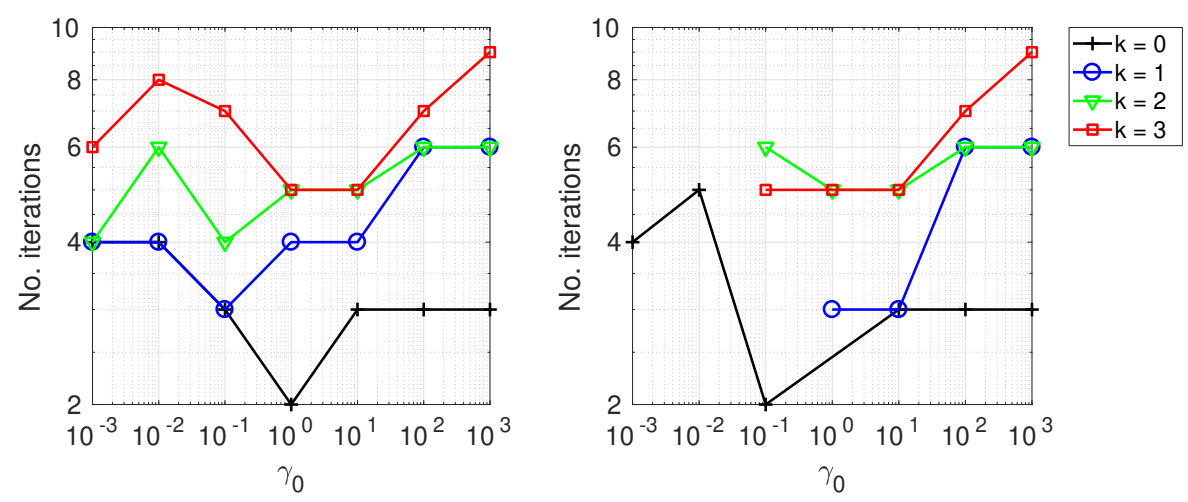

Figure 3: Influence of the penalty parameter $\gamma_{0}$ on the number of semi-smooth Newton iterations. Hexagonal mesh with mesh-size $h=0.622, \theta=-1$ (left) and $\theta=1$ (right), $k \in\{0,1,2,3\}$.

elements. We depict in Figure 4 our numerical solution, which matches (qualitatively) the one presented in [15]. We present $H^{1}$-errors and convergence rates in Table 9 with polynomials of order $k \in\{0,1,2\}$. Remark that for $k \in\{1,2\}$ the convergence rates are below the optimal value of $(k+1)$, owing to the limited regularity of the exact solution, which is expected to be in $H^{\frac{5}{2}-\varepsilon}(\Omega)$ in the neighborhood of $\Gamma_{C}[54]$.

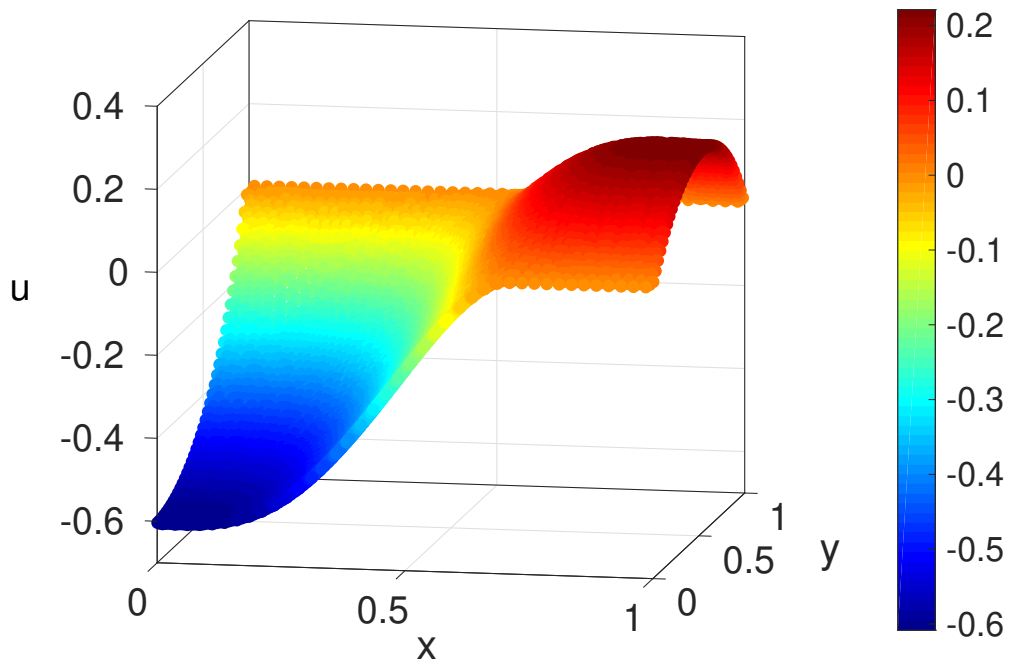

Figure 4: Computed numerical solution for Signorini conditions and test case 2.

Table 9: Test case 2 for Signorini conditions. $H^{1}$-errors and convergence rates. Cell version with $\theta=1$ and $\gamma_{0}=10$.

\begin{tabular}{lllllll}
\hline & \multicolumn{2}{c}{$k=0$} & \multicolumn{2}{c}{$k=1$} & \multicolumn{2}{c}{$k=2$} \\
\hline$h$ & error & rate & error & rate & error & rate \\
\hline 0.168 & $2.492 \mathrm{e}-01$ & & $3.734 \mathrm{e}-02$ & & $9.746 \mathrm{e}-03$ & \\
0.084 & $1.275 \mathrm{e}-01$ & 0.967 & $9.794 \mathrm{e}-03$ & 1.931 & $2.938 \mathrm{e}-03$ & 1.730 \\
0.042 & $6.445 \mathrm{e}-02$ & 0.984 & $2.666 \mathrm{e}-03$ & 1.877 & $1.143 \mathrm{e}-03$ & 1.361 \\
0.021 & $3.240 \mathrm{e}-02$ & 0.992 & $7.754 \mathrm{e}-04$ & 1.781 & $3.409 \mathrm{e}-04$ & 1.746 \\
\hline
\end{tabular}




\section{References}

[1] M. Abbas, A. Ern, and N. Pignet. Hybrid High-Order methods for finite deformations of hyperelastic materials. Comput. Mech., 62(4):909-928, 2018.

[2] M. Abbas, A. Ern, and N. Pignet. A Hybrid High-Order method for incremental associative plasticity with small deformations. Available at http://hal.archives-ouvertes.fr/hal-01768411, 2018.

[3] P. Alart. Méthode de Newton généralisée en mécanique du contact. J. Math. Pures Appl. (9), 76(1): 83-108, 1997.

[4] B. Ayuso de Dios, K. Lipnikov, and G. Manzini. The nonconforming virtual element method. ESAIM: Math. Model. Numer. Anal. (M2AN), 50(3):879-904, 2016.

[5] F. Ben Belgacem and Y. Renard. Hybrid finite element methods for the Signorini problem. Math. Comp., 72(243):1117-1145, 2003.

[6] S. Bertoluzza, M. Pennacchio, and D. Prada. High order VEM on curved domains. arXiv preprint arXiv:1811.04755, 2018.

[7] T. Boiveau and E. Burman. A penalty-free Nitsche method for the weak imposition of boundary conditions in compressible and incompressible elasticity. IMA J. Numer. Anal., 36(2):770-795, 2016.

[8] J. Bonelle and A. Ern. Analysis of compatible discrete operator schemes for elliptic problems on polyhedral meshes. ESAIM Math. Model. Numer. Anal., 48(2):553-581, 2014.

[9] M. Botti, D. A. Di Pietro, and P. Sochala. A Hybrid High-Order method for nonlinear elasticity. SIAM J. Numer. Anal., 55(6):2687-2717, 2017.

[10] H. Brezis. Équations et inéquations non linéaires dans les espaces vectoriels en dualité. Ann. Inst. Fourier (Grenoble), 18(fasc. 1):115-175, 1968.

[11] F. Brezzi, K. Lipnikov, and M. Shashkov. Convergence of the mimetic finite difference method for diffusion problems on polyhedral meshes. SIAM J. Numer. Anal., 43(5):1872-1896, 2005.

[12] E. Burman. A penalty-free nonsymmetric Nitsche-type method for the weak imposition of boundary conditions. SIAM J. Numer. Anal., 50(4):1959-1981, 2012.

[13] E. Burman and A. Ern. An unfitted Hybrid High-Order method for elliptic interface problems. SIAM J. Numer. Anal., 56(3):1525-1546, 2018.

[14] E. Burman, P. Hansbo, and M. G. Larson. The penalty-free Nitsche method and nonconforming finite elements for the Signorini problem. SIAM J. Numer. Anal., 55(6):2523-2539, 2017.

[15] E. Burman, P. Hansbo, and M. G. Larson. Augmented Lagrangian finite element methods for contact problems. ESAIM Math. Model. Numer. Anal., 53(1):173-195, 2019.

[16] K. L. Cascavita, J. Bleyer, X. Chateau, and A. Ern. Hybrid discretization methods with adaptive yield surface detection for Bingham pipe flows. J. Sci. Comput., 77(3):1424-1443, 2018.

[17] F. Chouly. An adaptation of Nitsche's method to the Tresca friction problem. J. Math. Anal. Appl., 411(1):329-339, 2014.

[18] F. Chouly and P. Hild. On convergence of the penalty method for unilateral contact problems. Appl. Numer. Math., 65:27-40, 2013.

[19] F. Chouly and P. Hild. A Nitsche-based method for unilateral contact problems: numerical analysis. SIAM J. Numer. Anal., 51(2):1295-1307, 2013.

[20] F. Chouly, P. Hild, and Y. Renard. Symmetric and non-symmetric variants of Nitsche's method for contact problems in elasticity: theory and numerical experiments. Math. Comp., 84(293):1089-1112, 2015. 
[21] F. Chouly, M. Fabre, P. Hild, R. Mlika, J. Pousin, and Y. Renard. An overview of recent results on Nitsche's method for contact problems. In Geometrically unfitted finite element methods and applications, volume 121 of Lect. Notes Comput. Sci. Eng., pages 93-141. Springer, Cham, 2017.

[22] P.-G. Ciarlet. The finite element method for elliptic problems, volume II of Handbook of Numerical Analysis (eds. P.G. Ciarlet and J.L. Lions). North-Holland Publishing Co., Amsterdam, 1991.

[23] M. Cicuttin, D. A. Di Pietro, and A. Ern. Implementation of Discontinuous Skeletal methods on arbitrary-dimensional, polytopal meshes using generic programming. J. Comput. Appl. Math., 344: 852-874, 2018.

[24] B. Cockburn, J. Gopalakrishnan, and R. Lazarov. Unified hybridization of discontinuous Galerkin, mixed, and continuous Galerkin methods for second order elliptic problems. SIAM J. Numer. Anal., 47(2):1319-1365, 2009.

[25] B. Cockburn, D. A. Di Pietro, and A. Ern. Bridging the Hybrid High-Order and Hybridizable discontinuous Galerkin methods. ESAIM Math. Model. Numer. Anal., 50(3):635-650, 2016.

[26] A. Curnier and P. Alart. A generalized Newton method for contact problems with friction. J. Méc. Théor. Appl., 7(suppl. 1):67-82, 1988.

[27] D. A. Di Pietro and J. Droniou. A Hybrid High-Order method for Leray-Lions elliptic equations on general meshes. Math. Comp., 86(307):2159-2191, 2017.

[28] D. A. Di Pietro and A. Ern. Mathematical aspects of discontinuous Galerkin methods, volume 69 of Mathématiques $\& 3$ Applications. Springer, Heidelberg, 2012.

[29] D. A. Di Pietro and A. Ern. A Hybrid High-Order locking-free method for linear elasticity on general meshes. Comput. Methods Appl. Mech. Engrg., 283:1-21, 2015.

[30] D. A. Di Pietro, A. Ern, and S. Lemaire. An arbitrary-order and compact-stencil discretization of diffusion on general meshes based on local reconstruction operators. Comput. Methods Appl. Math., 14 (4):461-472, 2014.

[31] D. A. Di Pietro, J. Droniou, and A. Ern. A discontinuous-skeletal method for advection-diffusionreaction on general meshes. SIAM J. Numer. Anal., 53(5):2135-2157, 2015.

[32] D. A. Di Pietro, A. Ern, A. Linke, and F. Schieweck. A discontinuous skeletal method for the viscositydependent Stokes problem. Comput. Methods Appl. Mech. Engrg., 306:175-195, 2016.

[33] J. K. Djoko. Discontinuous Galerkin finite element methods for variational inequalities of first and second kinds. Numer. Methods Partial Differential Equations, 24(1):296-311, 2008.

[34] J. Droniou, R. Eymard, T. Gallouët, and R. Herbin. A unified approach to mimetic finite difference, hybrid finite volume and mixed finite volume methods. Math. Models Methods Appl. Sci., 20(2):265295,2010 .

[35] G. Drouet and P. Hild. Optimal convergence for discrete variational inequalities modelling Signorini contact in 2D and 3D without additional assumptions on the unknown contact set. SIAM J. Numer. Anal., 53(3):1488-1507, 2015.

[36] A. Ern and J.-L. Guermond. Finite element quasi-interpolation and best approximation. ESAIM Math. Model. Numer. Anal., 51(4):1367-1385, 2017.

[37] R. Glowinski. Numerical methods for nonlinear variational problems. Springer-Verlag, 2008.

[38] R. Glowinski and P. Le Tallec. Augmented Lagrangian and operator-splitting methods in nonlinear mechanics, volume 9 of SIAM Studies in Applied Mathematics. Society for Industrial and Applied Mathematics (SIAM), Philadelphia, PA, 1989.

[39] Q. Guan, M. Gunzburger, and W. Zhao. Weak-Galerkin finite element methods for a second-order elliptic variational inequality. Comput. Methods Appl. Mech. Engrg., 337:677-688, 2018. 
[40] W. Han and M. Sofonea. Quasistatic contact problems in viscoelasticity and viscoplasticity, volume 30 of AMS/IP Studies in Advanced Mathematics. American Mathematical Society, Providence, RI, 2002.

[41] P. Hansbo. Nitsche's method for interface problems in computational mechanics. GAMM-Mitt., 28(2): 183-206, 2005.

[42] J. Haslinger. Finite element analysis for unilateral problems with obstacles on the boundary. Apl. Mat., 22(3):180-188, 1977.

[43] J. Haslinger, I. Hlaváček, and J. Nečas. Numerical methods for unilateral problems in solid mechanics, volume IV of Handbook of Numerical Analysis (eds. P.G. Ciarlet and J.L. Lions). North-Holland Publishing Co., Amsterdam, 1996.

[44] P. Hild. Numerical implementation of two nonconforming finite element methods for unilateral contact. Comput. Methods Appl. Mech. Engrg., 184(1):99-123, 2000.

[45] P. Hild and Y. Renard. A stabilized Lagrange multiplier method for the finite element approximation of contact problems in elastostatics. Numer. Math., 115(1):101-129, 2010.

[46] P. Hild and Y. Renard. An improved a priori error analysis for finite element approximations of Signorini's problem. SIAM J. Numer. Anal., 50(5):2400-2419, 2012.

[47] S. Hüeber and B. I. Wohlmuth. An optimal a priori error estimate for nonlinear multibody contact problems. SIAM J. Numer. Anal., 43(1):156-173, 2005.

[48] N. Kikuchi and J. T. Oden. Contact problems in elasticity: a study of variational inequalities and finite element methods, volume 8 of SIAM Studies in Applied Mathematics. Society for Industrial and Applied Mathematics (SIAM), Philadelphia, PA, 1988.

[49] K. Kunisch and G. Stadler. Generalized Newton methods for the 2D-Signorini contact problem with friction in function space. M2AN Math. Model. Numer. Anal., 39(4):827-854, 2005.

[50] Y. Kuznetsov, K. Lipnikov, and M. Shashkov. The mimetic finite difference method on polygonal meshes for diffusion-type problems. Comput. Geosci., 8(4):301-324 (2005), 2004.

[51] P. Laborde and Y. Renard. Fixed point strategies for elastostatic frictional contact problems. Math. Methods Appl. Sci., 31(4):415-441, 2008.

[52] T. A. Laursen. Computational contact and impact mechanics. Springer-Verlag, Berlin, 2002.

[53] C. Lehrenfeld. Hybrid Discontinuous Galerkin methods for solving incompressible flow problems. $\mathrm{PhD}$ thesis, Rheinisch-Westfälische Technische Hochschule Aachen, 2010.

[54] M. Moussaoui and K. Khodja. Régularité des solutions d'un problème mêlé Dirichlet-Signorini dans un domaine polygonal plan. Comm. Partial Differential Equations, 17(5-6):805-826, 1992.

[55] J. Nitsche. Über ein Variationsprinzip zur Lösung von Dirichlet-Problemen bei Verwendung von Teilräumen, die keinen Randbedingungen unterworfen sind. Abh. Math. Sem. Univ. Hamburg, 36:9-15, 1971.

[56] Y. Renard. Generalized Newton's methods for the approximation and resolution of frictional contact problems in elasticity. Comput. Methods Appl. Mech. Engrg., 256:38-55, 2012.

[57] B. Rivière, M. F. Wheeler, and V. Girault. A priori error estimates for finite element methods based on discontinuous approximation spaces for elliptic problems. SIAM J. Numer. Anal., 39(3):902-931, 2001.

[58] F. Scarpini and M.-A. Vivaldi. Error estimates for the approximation of some unilateral problems. RAIRO Anal. Numér., 11(2):197-208, 1977.

[59] R. Stenberg. On some techniques for approximating boundary conditions in the finite element method. J. Comput. Appl. Math., 63(1-3):139-148, 1995. 
[60] F. Wang and H. Wei. Virtual element method for simplified friction problem. Appl. Math. Letters, 85: $125-131,2018$.

[61] F. Wang and H. Wei. Virtual element methods for the obstacle problem. IMA J. Numer. Anal., 2019. To appear.

[62] F. Wang, W. Han, and X.-L. Cheng. Discontinuous Galerkin methods for solving elliptic variational inequalities. SIAM J. Numer. Anal., 48(2):708-733, 2010.

[63] F. Wang, W. Han, and X. Cheng. Discontinuous Galerkin methods for solving the Signorini problem. IMA J. Numer. Anal., 31(4):1754-1772, 2011.

[64] T. Warburton and J. S. Hesthaven. On the constants in $h p$-finite element trace inverse inequalities. Comput. Methods Appl. Mech. Engrg., 192(25):2765-2773, 2003.

[65] B. Wohlmuth. Variationally consistent discretization schemes and numerical algorithms for contact problems. Acta Numer., 20:569-734, 2011.

[66] P. Wriggers. Computational Contact Mechanics. Wiley, 2002.

[67] P. Wriggers, W. T. Rust, and B. D. Reddy. A virtual element method for contact. Comput. Mech., 58 (6):1039-1050, 2016.

[68] W. Xing, J. Zhang, C. Song, and F. Tin-Loi. A node-to-node scheme for three-dimensional contact problems using the scaled boundary finite element method. Comput. Methods Appl. Mech. Engrg., 347: 928-956, 2019.

[69] M. Zhao, H. Wu, and C. Xiong. Error analysis of HDG approximations for elliptic variational inequality: obstacle problem. Numer. Algorithms, 81(2):445-463, 2019. 